\title{
ASYMPTOTIC SOLUTIONS OF LINEAR ORDINARY DIFFERENTIAL EQUATIONS AT AN IRREGULAR SINGULARITY OF RANK UNITY
}

\author{
F. W. J. Olver
}

\begin{abstract}
New existence theorems are constructed for the asymptotic solutions of linear ordinary differential equations of arbitrary order in the neighborhood of an irregular singularity of rank 1 with distinct characteristic values. Advantages over existing results include more extensive regions of validity, explicit and realistic bounds for the remainder terms in the asymptotic expansions, and greater simplicity. In general the regions of validity are maximal.
\end{abstract}

\section{Introduction}

This investigation is aimed at homogeneous linear ordinary differential equations having an irregular singularity, which we may suppose without loss of generality to be located at the point at infinity in the complex plane $(\mathbb{C})$. We may represent these equations in the forms

$$
\begin{gathered}
L w \equiv \frac{d^{n} w}{d z^{n}}+f_{n-1}(z) \frac{d^{n-1} w}{d z^{n-1}}+f_{n-2}(z) \frac{d^{n-2} w}{d z^{n-2}}+\cdots+f_{0}(z) w=0, \\
\frac{d \mathbf{w}}{d z}=\mathbf{F}(z) \mathbf{w}
\end{gathered}
$$

for example. In (1.1), $n-2$ is an arbitrary nonnegative integer and the coefficients $f_{\ell}(z), \ell=0,1, \ldots, n-1$, are analytic in a punctured neighborhood of infinity. In (1.2), $\mathbf{w}$ is a column vector of length $n$ and $\mathbf{F}(z)$ is an $n \times n$ matrix whose elements are analytic in a punctured neighborhood of infinity; again $n \geq 2$. As is well known, an equation having either of the forms (1.1), (1.2) can be transformed into the other. ${ }^{1}$ Consequently, to any asymptotic theory applicable to one of these forms, there is a corresponding theory for the other. In our investigation, we concentrate on the scalar equation (1.1), partly because almost all recent literature for general $n$ applies to the vector form (1.2), but more importantly because the matrix approach has had the weakness of treating all asymptotic solutions as having the same character and enjoying only a common region of validity.

Without loss of generality, we shall suppose that the irregular singularity is located at the point at infinity. Let $r$ be the least nonnegative integer for which all of the functions $z^{(\ell-n)(r-1)} f_{\ell}(z)$ are analytic at $\infty$. When $r=0$, infinity is either an ordinary point or a regular singularity [7, Chapter 7]. We shall not be concerned with this case.

Received November 28, 1996, revised March 18, 1997.

1991 Mathematics Subject Classification: 34E05.

Key words and phrases: asymptotic expansions, canonical sectors, error bounds, LiouvilleNeumann series, remainder terms, variational operator, Volterra integral equation.

${ }^{1}$ More precisely, only special forms of (1.2) are equivalent to (1.1); conversely, each element of a solution vector of (1.2) satisfies a different form of $(1.1)$ in general [26, §1]. 
In other cases when $r$ exists, we say that $\infty$ is a singularity of the differential equation of rank $r$.

For $n=2$, there is a well-developed and frequently applied theory of asymptotic solutions, complete with error bounds; see, for example, [15], [16, Chapter 7], [18]. Also for $n=2$ and $r=\infty$ (that is, when at least one of the coefficients $f_{\ell}(z)$ has an essential singularity), the asymptotic forms of the solutions, again with error bounds, can be found by application of the Liouville-Green approximation theorems given in [16, Chapter 6]. By comparison, and as explained more fully in a companion paper [14], existing theories for higher values of $n$ are disappointing in some ways. Regions of validity are unnecessarily restricted, there is a lack of information about bounds for the remainder terms, and the differing natures of the asymptotic solutions are not revealed.

The purpose of the present paper is to supply a more comprehensive theory for arbitrary values of $n$ in the case $r=1$. New forms of asymptotic existence theorems are stated and proved, and explicit bounds for the remainder terms are constructed.

The paper is arranged as follows. In $\S 2$, we construct formal series solutions of (1.1) in descending powers of $z$, with the recurrence relation for the coefficients expressed in explicit form. The main asymptotic existence theorems are stated in $\S 3$. The proof of these theorems begins in $\S 4$ with the construction of an approximating differential equation with known solutions. In $\S 5$, we derive a singular Volterra integral equation that is satisfied by the remainder term associated with any chosen formal expansion. This equation is solved in a Liouville-Neumann series in $\S \S 6-8$, complete with upper bounds on the sum of the series. This analysis is an extension of that given in [16, Chapter 7] for the case $n=2$, but is considerably more difficult; it owes much to an earlier investigation of F. Stenger [19]. In $\S 9$, the bounds found in $\S \S 6-8$ for the remainder term are discussed and simplified (at a cost of some sharpness). The proof of the asymptotic existence theorems of $\S 3$ is completed in $\S 10$. Lastly, in $\S 11$, we discuss the extent of the regions of validity and the asymptotic nature of the error bounds.

\section{Formal solutions}

By hypothesis, the singularity of equation (1.1) at infinity is of rank 1. Hence the coefficients $f_{\ell}(z)$ can be expanded in power series

$$
f_{\ell}(z)=\sum_{s=0}^{\infty} \frac{f_{s \ell}}{z^{s}}, \quad \ell=0,1, \ldots, n-1,
$$

that converge on an open annulus $|z|>a$, say. We also can extend (2.1) to $\ell=n$, by defining $f_{0 n}=1$ and $f_{s n}=0(s \geq 1)$.

Formal solutions of (1.1) in descending powers of $z$ are the so-called normal solutions

$$
w(z)=e^{\lambda z} z^{\mu} \sum_{s=0}^{\infty} \frac{a_{s}}{z^{s}},
$$

in which $\lambda, \mu$, and the coefficients $a_{s}$ are constants. Formal differentiation yields

$$
w^{(\ell)}(z)=e^{\lambda z} z^{\mu} \sum_{s=0}^{\infty} \frac{a_{s}^{(\ell)}}{z^{s}}, \quad \ell=1,2, \ldots
$$


where

$$
a_{s}^{(\ell)}=\lambda a_{s}^{(\ell-1)}+(\mu-s+1) a_{s-1}^{(\ell-1)},
$$

with the understanding that $a_{s}^{(0)}=a_{s}$ and coefficients of negative suffix vanish. By induction,

$$
a_{s}^{(\ell)}=\sum_{p=0}^{\ell}\left(\begin{array}{l}
\ell \\
p
\end{array}\right) \lambda^{\ell-p}(\mu-s+1)_{p} a_{s-p}
$$

where $(\mu-s+1)_{p}$ is the usual Pochhammer symbol for the ascending factorial $(\mu-s+1)(\mu-s+2) \cdots(\mu-s+p)$.

On substitution into (1.1) by means of (2.1), (2.2), and (2.3) and equating coefficients of $e^{\lambda z} z^{\mu-s}$, we obtain

$$
\sum_{\ell=0}^{n} \sum_{k=0}^{s} f_{k \ell} a_{s-k}^{(\ell)}=0 .
$$

Then by substituting into this equation using (2.4) and interchanging the order of summation, we find that

$$
\sum_{p=0}^{n} \sum_{k=0}^{s-p}\left(\begin{array}{c}
\mu-s+k+p \\
p
\end{array}\right) \phi_{k}^{(p)}(\lambda) a_{s-k-p}=0
$$

where $\phi_{k}^{(p)}(\lambda)$ denotes the $p$ th derivative of the polynomial

$$
\phi_{k}(\lambda)=\sum_{\ell=0}^{n} f_{k \ell} \lambda^{\ell}
$$

If we now replace $k$ by $k-p$ in (2.5) and again interchange the order of summation, we obtain

$$
\sum_{k=0}^{s} a_{s-k} \sum_{p=0}^{\min (k, n)}\left(\begin{array}{c}
\mu-s+k \\
p
\end{array}\right) \phi_{k-p}^{(p)}(\lambda)=0 .
$$

Without loss of generality, set $a_{0}=1$. Then with $s=0$, equation (2.7) reduces to the characteristic equation

$$
\phi_{0}(\lambda)=0
$$

Next, on taking $s=1$ in (2.7) and using (2.8), we obtain the indicial equation

$$
\mu \phi_{0}^{\prime}(\lambda)+\phi_{1}(\lambda)=0 \text {. }
$$

For higher values of $s$, we note that as a consequence of (2.8), the lower limit in the outer sum in (2.7) may be replaced by unity. Then changing $s$ and $k$ into $s+1$ and $k+1$, respectively, solving for $a_{s}$, and using (2.9), we arrive at

$$
s \phi_{0}^{\prime}(\lambda) a_{s}=\sum_{k=1}^{s} a_{s-k} \sum_{p=0}^{\min (k+1, n)}\left(\begin{array}{c}
\mu-s+k \\
p
\end{array}\right) \phi_{k+1-p}^{(p)}(\lambda), \quad s \geq 1 .
$$

The $n$ roots $\lambda_{1}, \lambda_{2}, \ldots, \lambda_{n}$, say, of (2.8) are the characteristic values. Equation (2.9) yields the corresponding values $\mu_{1}, \mu_{2}, \ldots, \mu_{n}$, say, of $\mu$, the indices or exponents. 
Lastly, equation (2.10) determines the corresponding coefficients $a_{s 1}, a_{s 2}, \ldots, a_{s n}$, say, for $s=1,2, \ldots$ In this way, we have $n$ formal solutions

$$
w_{j}(z)=e^{\lambda_{j} z} z^{\mu_{j}} \sum_{s=0}^{\infty} \frac{a_{s j}}{z^{s}}, \quad j=1,2, \ldots, n,
$$

each being normalized by the condition $a_{0 j}=1$. As is well known, the process fails if, and only if, $\phi_{0}^{\prime}(\lambda)=0$, that is, if the characteristic equation has multiple roots. We shall not pursue these cases in the present paper. ${ }^{2}$

Owing to the presence of the binomial coefficients on the right-hand side of (2.10), the coefficients $a_{s}$ will usually grow as factorials as $s \rightarrow \infty$; compare [17]. In consequence, the formal series (2.11) diverge in general.

\section{Main theorems}

The sectors of validity of the asymptotic solutions that correspond to the formal solutions (2.11) depend on the configuration of the $\lambda$ 's in $\mathbb{C}$. We introduce the following definitions. Write

$$
\theta_{j k}=\operatorname{ph}\left(\lambda_{k}-\lambda_{j}\right), \quad k \neq j .
$$

To remove the ambiguities in the choice of these angles, let $\mathcal{J}$ be any prescribed halfclosed interval of length $2 \pi$-the closure may be on the left or on the right. The unique set of the $n(n-1)$ angles $\theta_{j k}$ that belong to J we call the canonical set of the $\operatorname{ph}\left(\lambda_{k}-\lambda_{j}\right)$ corresponding to J. Next, for each $j$, let

$$
\alpha_{j}=\min _{k \neq j} \theta_{j k}, \quad \beta_{j}=\max _{k \neq j} \theta_{j k} .
$$

Then

$$
0 \leq \beta_{j}-\alpha_{j}<2 \pi
$$

We call the open sector

$$
\mathcal{S}_{j}(\mathcal{J})=\left(z:-\frac{3}{2} \pi-\alpha_{j}<\operatorname{ph} z<\frac{3}{2} \pi-\beta_{j}\right)
$$

the canonical $j$-sector corresponding to J. As a consequence of (3.3), the angle of $\mathcal{S}_{j}(\mathcal{J})$ lies in the half-closed interval $(\pi, 3 \pi]$. Thus, $\mathcal{S}_{j}(\mathcal{J})$ exceeds half a Riemann sheet, but cannot exceed one and a half Riemann sheets.

In a similar manner, let

$$
\alpha=\min _{k \neq j} \theta_{j k}, \quad \beta=\max _{k \neq j} \theta_{j k},
$$

the minimum and maximum now being taken over all possible values of $j$ and $k$. Then

$$
\pi \leq \beta-\alpha<2 \pi \text {. }
$$

(The left-hand inequality follows from the fact that in a canonical set, $\theta_{k j}$ is either $\theta_{j k}+\pi$ or $\theta_{j k}-\pi$.) We call the open sector

$$
\mathcal{S}(\mathcal{J})=\left(z:-\frac{3}{2} \pi-\alpha<\operatorname{ph} z<\frac{3}{2} \pi-\beta\right)
$$

\footnotetext{
${ }^{2} \mathrm{An}$ approach for handling these exceptional cases is to make a simple change of variable, for example, $z=\zeta^{2}$ or other power of $\zeta$. For general formal solutions when the characteristic equation has multiple roots, see [20], [22], [24] and [26, Chapter 5].
} 
the canonical sector corresponding to J. Again, as a consequence of $(3.6), \mathcal{S}(\mathcal{J})$ exceeds half a Riemann sheet, but cannot exceed one Riemann sheet. It also is evident that ${ }^{3}$

$$
\mathcal{S}(\mathcal{J})=\mathcal{S}_{1}(\mathcal{J}) \cap \mathcal{S}_{2}(\mathcal{J}) \cap \cdots \cap \mathcal{S}_{n}(\mathcal{J}) .
$$

Theorem 3.1. For a given interval $\mathcal{J}$ and for each $j=1,2, \ldots, n$, there exists a unique solution $w_{j}(\mathcal{J} \mid z)$ of $(1.1)$ such that

$$
w_{j}(\mathcal{J} \mid z) \sim e^{\lambda_{j} z} z^{\mu_{j}} \sum_{s=0}^{\infty} \frac{a_{s j}}{z^{s}}
$$

as $z \rightarrow \infty$, uniformly in any closed sector properly interior to $\mathcal{S}_{j}(\mathcal{J})$. Furthermore, this asymptotic expansion can be differentiated $n-1$ times in the same circumstances, and the $n$ solutions $w_{j}(\mathcal{J} \mid z)$ are linearly independent.

Theorem 3.2. In any canonical sector $\mathcal{S}(\mathcal{J})$, there exist linearly independent and unique solutions $w_{j}(\mathcal{J} \mid z), j=1,2, \ldots, n$, such that (3.9) applies for each $j$ as $z \rightarrow \infty$ uniformly in any closed sector properly interior to $\mathcal{S}(\mathcal{J})$.

Theorems 3.1 and 3.2 are proved in $\S \S 4-10$, and in the process, we shall construct bounds for the remainder terms in the asymptotic expansions and their differentiated forms. We observe here that except for the uniqueness property pertaining to the common sector $\mathcal{S}(\mathcal{J})$, Theorem 3.2 follows immediately from Theorem 3.1. Conversely, Theorem 3.1 could be deduced from Theorem 3.2 by repeated use of connection formulas; however, this approach does not yield bounds for the remainder terms.

The special case of these theorems when $n=2$ is well known; see, for example, [16, Chapter 7]. For higher values of $n$, the existing literature supplies results that are essentially equivalent either to Theorem 3.2, e.g., [2], [3], or to forms of Theorem 3.2 that are weaker insofar as the region of validity and uniqueness property are concerned, e.g., [5], [22], [26]. ${ }^{4}$ See also the surveys included in [8], [25]. As suggested in $\S 1$, this emphasis on a common region of validity for all solutions rather than separate regions for individual solutions may well be a consequence of the matrix approach to systems of equations. However, as demonstrated in [14], the increased regions of validity furnished by the stronger result, Theorem 3.1, are very important in applications, including the computation of solutions outside the neighborhood of infinity.

\section{Differential equation satisfied by leading terms}

Three general methods are available to prove Theorems 3.1 and 3.2. The first is to seek integral representations of the solutions in the form of generalized Laplace transforms. This approach was used, for example, by Birkhoff [3], and it also underlies more recent developments based upon summation of the formal divergent series by Borel-Laplace transforms, for example, [1], [11], [21]. The second method is to solve an integral equation satisfied by the remainder term in the asymptotic expansion. This is used, for example, in [5, Chapter 5], [16, Chapter 7], [19], [20], [26, Chapters 4 and 5]. Lastly, convergent expansions in series of inverse factorials can be constructed for the solutions, see, for example, [23], [24]. We shall adopt the second method because of its

\footnotetext{
${ }^{3}$ It can happen that $\mathcal{S}_{j}(\mathcal{J})=\mathcal{S}(\mathcal{J})$. This is the case when all the $\lambda$ s are collinear, and $\lambda_{j}$ is not an endpoint of the line.

${ }^{4}$ An important exception is the work of Stenger [19], [20]. These papers seem to have been overlooked by later writers.
} 
directness, and also because it lends itself more readily to the construction of bounds for the remainder term.

As a preliminary step, we need to construct an approximating differential equation with known solutions. For this purpose, we derive an equation $\widehat{L} w=0$ of the form (1.1) that is satisfied exactly by the functions

$$
\widehat{w}_{j}(z)=e^{\lambda_{j} z} z^{\mu_{j}}, \quad j=1,2, \ldots, n,
$$

where $\lambda_{j}$ and $\mu_{j}$ are determined as in $\S 2$.

One way to proceed is as follows. ${ }^{5}$ Let $\widehat{L}_{m} w=0$ be a linear differential equation of order $m$ satisfied by $\widehat{w}_{1}(z), \widehat{w}_{2}(z), \ldots, \widehat{w}_{m}(z)$. Then

$$
\widehat{L}_{m+1} w=e^{-\lambda_{m+1} z} z^{-\mu_{m+1}}\left\{\left(\widehat{L}_{m} \widehat{w}_{m+1}\right) \frac{d}{d z}\left(\widehat{L}_{m} w\right)-\left(\widehat{L}_{m} w\right) \frac{d}{d z}\left(\widehat{L}_{m} \widehat{w}_{m+1}\right)\right\} \text {. }
$$

Beginning with

$$
\widehat{L}_{1}=\frac{d}{d z}-\left(\lambda_{1}+\frac{\mu_{1}}{z}\right),
$$

we can calculate the operators $\widehat{L}_{2}, \widehat{L}_{3}, \ldots$, by successive application of (4.2) until $\widehat{L}_{n}$ is obtained. Then dividing $\widehat{L}_{n}$ by its coefficient of $(d / d z)^{n}$, we arrive at the desired operator $\widehat{L}$ in the form

$$
\widehat{L}=\left(\frac{d}{d z}\right)^{n}+\widehat{f}_{n-1}(z)\left(\frac{d}{d z}\right)^{n-1}+\cdots+\widehat{f}_{0}(z) .
$$

The coefficients $\widehat{f}_{\ell}(z), \ell=0,1, \ldots, n-1$, are rational functions of $z$ that can be expanded in series

$$
\widehat{f}_{\ell}(z)=\sum_{s=0}^{\infty} \frac{\widehat{f}_{s \ell}}{z^{s}}, \quad \ell=0,1, \ldots, n-1,
$$

convergent on an annulus $|z|>\widehat{a}$, say.

An alternative procedure that leads to a direct recurrence relation for generating the coefficients $\widehat{f}_{s \ell}$ is as follows. On referring to equations (2.8) and (2.9), we see that the polynomials $\phi_{0}(\lambda)$ and $\phi_{1}(\lambda)$, corresponding to the desired equation $\widehat{L} w=0$, have to be the same as before. As a consequence, in the expansions (4.4), we have

$$
\widehat{f}_{0 \ell}=f_{0 \ell}, \quad \widehat{f}_{1 \ell}=f_{1 \ell}, \quad \ell=0,1, \ldots, n-1 .
$$

To determine the remaining coefficients $\widehat{f}_{s} \ell$, we start from the equations $\widehat{L} w=0$ and (4.4), and retrace the analysis of $\S 2$, distinguishing corresponding quantities by the addition of hats when they differ from the originals. For example, from (2.10), we derive

$$
s \phi_{0}^{\prime}(\lambda) \widehat{a}_{s}=\sum_{k=1}^{s} \widehat{a}_{s-k} \sum_{p=0}^{\min (k+1, n)}\left(\begin{array}{c}
\mu-s+k \\
p
\end{array}\right) \widehat{\phi}_{k+1-p}^{(p)}(\lambda) .
$$

Clearly, our objective is achieved if we can arrange that $\widehat{a}_{s}=0$ when $\lambda=\lambda_{j}, \mu=\mu_{j}$, $j=1,2, \ldots, n$, and $s \geq 1$.

\footnotetext{
${ }^{5}$ Suggested to the author by A. B. Olde Daalhuis. T. M. Dunster [6] gives the result in the case $n=2$.
} 
On setting $\widehat{a}_{1}=0$ and bearing in mind that $\widehat{a}_{0}=a_{0}=1$, we have

$$
\widehat{\phi}_{2}(\lambda)+\left(\begin{array}{c}
\mu \\
1
\end{array}\right) \phi_{1}^{\prime}(\lambda)+\left(\begin{array}{l}
\mu \\
2
\end{array}\right) \phi_{0}^{\prime \prime}(\lambda)=0
$$

that is,

$$
\sum_{\ell=0}^{n-1} \widehat{f}_{2 \ell} \lambda^{\ell}+\left(\begin{array}{c}
\mu \\
1
\end{array}\right) \sum_{\ell=1}^{n-1} f_{1 \ell} \ell \lambda^{\ell-1}+\left(\begin{array}{c}
\mu \\
2
\end{array}\right) \sum_{\ell=2}^{n-1} f_{0 \ell} \ell(\ell-1) \lambda^{\ell-2}+\left(\begin{array}{l}
\mu \\
2
\end{array}\right) n(n-1) \lambda^{n-2}=0
$$

The condition that this equation is satisfied for $\lambda=\lambda_{j}, \mu=\mu_{j}, j=1,2, \ldots, n$, is expressed conveniently in matrix notation:

$$
\mathbf{\Lambda} \widehat{\mathbf{F}}_{2}+\mathbf{M}_{1} \frac{\partial \boldsymbol{\Lambda}}{\partial \lambda} \dot{\mathbf{F}}_{1}+\mathbf{M}_{2} \frac{\partial^{2} \boldsymbol{\Lambda}}{\partial \lambda^{2}} \mathbf{F}_{0}+\mathbf{M}_{2} \frac{\partial^{2} \boldsymbol{\lambda}}{\partial \lambda^{2}}=\mathbf{0}
$$

where

$$
\begin{gathered}
\boldsymbol{\Lambda}=\left[\begin{array}{cccc}
1 & \lambda_{1} & \cdots & \lambda_{1}^{n-1} \\
1 & \lambda_{2} & \cdots & \lambda_{2}^{n-1} \\
\vdots & \vdots & \vdots & \vdots \\
1 & \lambda_{n} & \cdots & \lambda_{n}^{n-1}
\end{array}\right] \\
\boldsymbol{\lambda}=\left[\lambda_{1}^{n}, \lambda_{2}^{n}, \ldots, \lambda_{n}^{n}\right]^{T}, \quad \mathbf{M}_{p}=\operatorname{diag}\left[\left(\begin{array}{c}
\mu_{1} \\
p
\end{array}\right),\left(\begin{array}{c}
\mu_{2} \\
p
\end{array}\right), \ldots,\left(\begin{array}{c}
\mu_{n} \\
p
\end{array}\right)\right] \\
\mathbf{F}_{k}=\left[f_{k 0}, f_{k 1}, \ldots, f_{k, n-1}\right]^{T}, \quad \widehat{\mathbf{F}}_{k}=\left[\widehat{f}_{k 0}, \widehat{f}_{k 1}, \ldots, \widehat{f}_{k, n-1}\right]^{T},
\end{gathered}
$$

and

$$
\frac{\partial}{\partial \lambda}=\frac{\partial}{\partial \lambda_{1}}+\frac{\partial}{\partial \lambda_{2}}+\cdots+\frac{\partial}{\partial \lambda_{n}}
$$

Since

$$
\operatorname{det} \boldsymbol{\Lambda}=\prod_{k>j}\left(\lambda_{k}-\lambda_{j}\right) \neq 0
$$

by hypothesis, equation (4.6) determines $\widehat{\mathbf{F}}_{2}$, and hence its elements $\widehat{f}_{20}, \widehat{f}_{21}, \ldots$, $\widehat{f}_{2, n-1}$, finitely and uniquely.

The process may be continued, setting $\widehat{a}_{2}=0, \widehat{a}_{3}=0, \ldots$, in turn. By induction, we may verify that

$$
\begin{aligned}
& \mathbf{\Lambda} \widehat{\mathbf{F}}_{s+1}+\mathbf{M}_{1} \frac{\partial \boldsymbol{\Lambda}}{\partial \lambda} \widehat{\mathbf{F}}_{s}+\mathbf{M}_{2} \frac{\partial^{2} \boldsymbol{\Lambda}}{\partial \lambda^{2}} \widehat{\mathbf{F}}_{s-1}+\cdots+\mathbf{M}_{s-1} \frac{\partial^{s-1} \boldsymbol{\Lambda}}{\partial \lambda^{s-1}} \widehat{\mathbf{F}}_{2} \\
& \quad+\mathbf{M}_{s} \frac{\partial^{s} \boldsymbol{\Lambda}}{\partial \lambda^{s}} \mathbf{F}_{1}+\mathbf{M}_{s+1} \frac{\partial^{s+1} \boldsymbol{\Lambda}}{\partial \lambda^{s+1}} \mathbf{F}_{0}+\mathbf{M}_{s+1} \frac{\partial^{s+1} \lambda}{\partial \lambda^{s+1}}=\mathbf{0}, \quad 1 \leq s \leq n-1
\end{aligned}
$$

and

$$
\mathbf{\Lambda} \widehat{\mathbf{F}}_{s+1}+\mathbf{M}_{1} \frac{\partial \boldsymbol{\Lambda}}{\partial \lambda} \widehat{\mathbf{F}}_{s}+\cdots+\mathbf{M}_{n-1} \frac{\partial^{n-1} \boldsymbol{\Lambda}}{\partial \lambda^{n-1}} \widehat{\mathbf{F}}_{s+2-n}=\mathbf{0}, \quad s \geq n .
$$

These equations enable each of the vectors $\widehat{\mathbf{F}}_{s}, s=2,3 \ldots$, to be found finitely and uniquely by recurrence, with the recurrence relation involving no more than $n$ consecutive $\widehat{\mathbf{F}}$ 's. 


\section{Integral equation for the remainder term}

Let $S$ be an arbitrary positive integer. From (1.1) and (2.1), we see that the function

$$
e^{-\lambda z} z^{-\mu} L\left\{e^{\lambda z} z^{\mu} \sum_{s=0}^{S-1} \frac{a_{s}}{z^{s}}\right\}
$$

is analytic on the annulus $|z|>a$. Furthermore, by referring to (2.7) and recalling that the lower limit in the outer sum can be changed to $k=1$, we see that in the expansion of the function (5.1) in descending powers of $z$, the coefficients of $1, z^{-1}, \ldots, z^{-S}$ all vanish, and that of $z^{-S-1}$ is given by the right-hand side of (2.10) with $s=S$. Hence,

$$
L\left\{e^{\lambda_{j} z} z^{\mu_{j}} \sum_{s=0}^{S-1} \frac{a_{s j}}{z^{s}}\right\}=e^{\lambda_{j} z} z^{\mu_{j}}\left\{\frac{S \phi_{0}^{\prime}\left(\lambda_{j}\right) a_{S j}}{z^{S+1}}+O\left(\frac{1}{z^{S+2}}\right)\right\}
$$

as $z \rightarrow \infty$, uniformly $\forall$ ph $z$.

Let us write

$$
w(z)=e^{\lambda_{j} z} z^{\mu_{j}} \sum_{s=0}^{S-1} \frac{a_{s j}}{z^{s}}+R_{S j}(z)
$$

where $w(z)$ is assumed to be a solution of (1.1). Then,

$$
L\left\{R_{S j}(z)\right\}=-e^{\lambda_{j} z} z^{\mu_{j}} T_{S j}(z)
$$

where $T_{S j}(z)$ is the content of the braces on the right-hand side of (5.2). Thus $T_{S j}(z)$ is analytic on the annulus $|z|>a$, and $T_{S j}(z)=O\left(z^{-S-1}\right)$ as $z \rightarrow \infty$. Our main objective is to show that with the right choice of $w(z)$ in (5.3), the operator $L$ can be inverted, and hence that $R_{S j}(z)=e^{\lambda_{j} z} z^{\mu_{j}} O\left(z^{-S}\right)$ as $z \rightarrow \infty$ under suitable restrictions in $\mathbb{C}$. We shall achieve this goal by a method similar to that used in [15], [19].

We compare the given equation $L w=0$ with $\widehat{L} w=0$, defined in $\S 4$, with the known solutions (4.1). We also shall write

$$
L=\widehat{L}+L_{2}
$$

so that $L_{2}$ is the operator

$$
L_{2}=\sum_{\ell=0}^{n-1}\left\{f_{\ell}(z)-\widehat{f}_{\ell}(z)\right\}\left(\frac{d}{d z}\right)^{\ell} .
$$

Using the decomposition (5.5) and applying the method of variation of parameters $[4, \S 8]$, we see that there is a solution of (5.4) that satisfies the singular Volterra integral equation ${ }^{6}$

$$
\begin{aligned}
R_{S j}(z)= & \sum_{k=1}^{n} \widehat{w}_{k}(z) \int_{Q_{k}(z)} \frac{\widehat{W}_{k}(t)}{\widehat{W}(t)} L_{2}\left\{R_{S j}(t)\right\} d t \\
& +\sum_{k=1}^{n} \widehat{w}_{k}(z) \int_{Q_{k}(z)} \frac{\widehat{W}_{k}(t)}{\widehat{W}(t)} e^{\lambda_{j} t} t^{\mu_{j}} T_{S j}(t) d t .
\end{aligned}
$$

\footnotetext{
${ }^{6}$ This may be verified directly with the aid of the identities (5.13) below.
} 
Here $\widehat{W}(z)$ denotes the Wronskian determinant

$$
\widehat{W}(z)=\left|\begin{array}{cccc}
\widehat{w}_{1}(z) & \widehat{w}_{2}(z) & \cdots & \widehat{w}_{n}(z) \\
\widehat{w}_{1}^{\prime}(z) & \widehat{w}_{2}^{\prime}(z) & \cdots & \widehat{w}_{n}^{\prime}(z) \\
\vdots & \vdots & \vdots & \vdots \\
\widehat{w}_{1}^{(n-1)}(z) & \widehat{w}_{2}^{(n-1)}(z) & \cdots & \widehat{w}_{n}^{(n-1)}(z)
\end{array}\right|
$$

and $\widehat{W}_{k}(z)$ is the determinant obtained by replacing the $k$ th column of $\widehat{W}(z)$ by $[0,0, \ldots, 0,1]^{T} ;$ thus

$$
\widehat{W}_{k}(z)=\left|\begin{array}{ccccccc}
\widehat{w}_{1}(z) & \cdots & \widehat{w}_{k-1}(z) & 0 & \widehat{w}_{k+1}(z) & \cdots & \widehat{w}_{n}(z) \\
\vdots & \vdots & \vdots & \vdots & \vdots & \vdots & \vdots \\
\widehat{w}_{1}^{(n-2)}(z) & \cdots & \widehat{w}_{k-1}^{(n-2)}(z) & 0 & \widehat{w}_{k+1}^{(n-2)}(z) & \cdots & \widehat{w}_{n}^{(n-2)}(z) \\
\widehat{w}_{1}^{(n-1)}(z) & \cdots & \widehat{w}_{k-1}^{(n-1)}(z) & 1 & \widehat{w}_{k+1}^{(n-1)}(z) & \cdots & \widehat{w}_{n}^{(n-1)}(z)
\end{array}\right| .
$$

Lastly, the integration path $Q_{k}(z)$ runs from $t=z$ to $t=\infty \exp \left(-i \theta_{j k}\right)$ where $\theta_{j k}$ belongs to the canonical set introduced in $\S 3$ when $k \neq j$, and $\theta_{j j}=\frac{1}{2}\left(\alpha_{j}+\beta_{j}\right)$. We assume that $z$ and $Q_{k}(z)$ lie in the intersection of the canonical $j$-sector $\mathcal{S}_{j}(\mathcal{J})$ with the annuli $|z|>a,|z|>\widehat{a}$. Furthermore, we assume that the branches of ph $t$ used in forming the fractional powers $t^{\mu_{1}}, t^{\mu_{2}}, \ldots, t^{\mu_{n}}$ are continuous on $Q_{k}(z)$.

We shall solve equation (5.7) by the standard procedure for constructing a LiouvilleNeumann series expansion

$$
R_{S j}(z)=\sum_{s=0}^{\infty} h_{s}(z)
$$

by the method of successive approximations. Subsequently, we shall verify directly that this series converges, and that its sum is a solution of the inhomogeneous differential equation (5.4).

We define the functions $h_{s}(z), s=0,1, \ldots$, by $^{7}$

$$
h_{0}(z)=\sum_{k=1}^{n} \widehat{w}_{k}(z) \int_{Q_{k}(z)} \frac{\widehat{W}_{k}(t)}{\widehat{W}(t)} e^{\lambda_{j} t} t^{\mu_{j}} T_{S j}(t) d t
$$

and

$$
h_{s}(z)=\sum_{k=1}^{n} \widehat{w}_{k}(z) \int_{Q_{k}(z)} \frac{\widehat{W}_{k}(t)}{\widehat{W}(t)} L_{2}\left\{h_{s-1}(t)\right\} d t, \quad s \geq 1 .
$$

We observe that if we replace the last row of the determinant $(5.8)$ by $\left[\widehat{w}_{1}^{(\ell)}(z)\right.$, $\left.\widehat{w}_{2}^{(\ell)}(z), \ldots, \widehat{w}_{n}^{(\ell)}(z)\right]$ and then expand along this row, we obtain the identities

$$
\sum_{k=1}^{n} \widehat{w}_{k}^{(\ell)}(z) \widehat{W}_{k}(z)= \begin{cases}0, & \ell=0,1, \ldots, n-2 \\ \widehat{W}(z), & \ell=n-1\end{cases}
$$

\footnotetext{
${ }^{7}$ For simplicity, we have not indicated the dependence of the functions $h_{s}(z)$ on $S$ and $j$ in the notation. We also are assuming that the integrals in (5.11), (5.12), and subsequent equations in this section all converge. This is confirmed later.
} 
Hence, on differentiating (5.11) and (5.12), we have, for $\ell=0,1, \ldots, n-1$,

$$
\begin{gathered}
h_{0}^{(\ell)}(z)=\sum_{k=1}^{n} \widehat{w}_{k}^{(\ell)}(z) \int_{Q_{k}(z)} \frac{\widehat{W}_{k}(t)}{\widehat{W}(t)} e^{\lambda_{j} t} t^{\mu_{j}} T_{S j}(t) d t, \\
h_{s}^{(\ell)}(z)=\sum_{k=1}^{n} \widehat{w}_{k}^{(\ell)}(z) \int_{Q_{k}(z)} \frac{\widehat{W}_{k}(t)}{\widehat{W}(t)} L_{2}\left\{h_{s-1}(t)\right\} d t, \quad s \geq 1,
\end{gathered}
$$

and for $\ell=n$,

$$
\begin{gathered}
h_{0}^{(n)}(z)=\sum_{k=1}^{n} \widehat{w}_{k}^{(n)}(z) \int_{Q_{k}(z)} \frac{\widehat{W}_{k}(t)}{\widehat{W}(t)} e^{\lambda_{j} t} t^{\mu_{j}} T_{S j}(t) d t-e^{\lambda_{j} z} z^{\mu_{j}} T_{S j}(z), \\
h_{s}^{(n)}(z)=\sum_{k=1}^{n} \widehat{w}_{k}^{(n)}(z) \int_{Q_{k}(z)} \frac{\widehat{W}_{k}(t)}{\widehat{W}(t)} L_{2}\left\{h_{s-1}(t)\right\} d t-L_{2}\left\{h_{s-1}(z)\right\}, s \geq 1 .
\end{gathered}
$$

From (5.14) and (5.15) it follows that

$$
\begin{gathered}
L_{2}\left\{h_{0}(z)\right\}=\sum_{k=1}^{n} L_{2}\left\{\widehat{w}_{k}(z)\right\} \int_{Q_{k}(z)} \frac{\widehat{W}_{k}(t)}{\widehat{W}(t)} e^{\lambda_{j} t} t^{\mu_{j}} T_{S j}(t) d t, \\
L_{2}\left\{h_{s}(z)\right\}=\sum_{k=1}^{n} L_{2}\left\{\widehat{w}_{k}(z)\right\} \int_{Q_{k}(z)} \frac{\widehat{W}_{k}(t)}{\widehat{W}(t)} L_{2}\left\{h_{s-1}(t)\right\} d t, \quad s \geq 1 .
\end{gathered}
$$

\section{Further preliminaries}

In order to bound the functions $h_{s}(z)$ introduced in $\S 5$, and their derivatives, we need upper bounds for the functions that appear on the right-hand sides of equations (5.14) to (5.19), and also further restrictions on the integration paths $Q_{k}(z)$. Let $b$ be an arbitrarily chosen constant that exceeds the quantities $a$ and $\widehat{a}$ introduced in $\S \S 2$ and 4 . Then there exists a finite constant $B_{S j}$ such that

$$
\left|T_{S j}(t)\right| \leq S B_{S j}|t|^{-S-1}
$$

when $|t| \geq b$.

Next, for $\ell=0,1, \ldots, n-1$, we have

$$
\widehat{w}_{k}^{(\ell)}(z)=e^{\lambda_{k} z} z^{\mu_{k}}\left\{\lambda_{k}^{\ell}+O\left(z^{-1}\right)\right\}, \quad z \rightarrow \infty
$$

(with the conventional understanding that $\lambda_{k}^{\ell}=1$ when $\lambda_{k}=\ell=0$ ). Hence

$$
\left|\widehat{w}_{k}^{(\ell)}(z)\right| \leq D_{k \ell}\left|e^{\lambda_{k} z} z^{\mu_{k}}\right|, \quad|z| \geq b
$$

where $D_{k \ell}$ is an assignable finite constant. Since, also

$$
f_{\ell}(z)-\widehat{f}_{\ell}(z)=O\left(z^{-2}\right), \quad z \rightarrow \infty,
$$

(compare (2.1), (4.4), and (4.5)), it follows that

$$
L_{2}\left\{\widehat{w}_{k}(z)\right\}=e^{\lambda_{k} z} z^{\mu_{k}-2} O(1), \quad z \rightarrow \infty .
$$

Therefore, there is another finite constant $G_{k}$ such that ${ }^{8}$

$$
\left|L_{2}\left\{\widehat{w}_{k}(z)\right\}\right| \leq G_{k}\left|e^{\lambda_{k} z} z^{\mu_{k}-2}\right|, \quad|z| \geq b .
$$

\footnotetext{
${ }^{8} \mathrm{An}$ upper bound for $G_{k}$ can be calculated with the aid of (6.3), (4.12), (4.13), and the use of vector and subordinate matrix norms [27, page 56].
} 
For the determinants $\widehat{W}(t)$ and $\widehat{W}_{k}(t)$, we have from $(4.7),(4.11),(5.8),(5.9)$, and (6.2)

$$
\widehat{W}(t)=e^{\left(\lambda_{1}+\lambda_{2}+\cdots+\lambda_{n}\right) t} t^{\mu_{1}+\mu_{2}+\cdots+\mu_{n}}\left\{\operatorname{det} \boldsymbol{\Lambda}+O\left(t^{-1}\right)\right\}, \quad t \rightarrow \infty
$$

and

$$
\widehat{W}_{k}(t)=C_{k} e^{-\lambda_{k} t} t^{-\mu_{k}} \widehat{W}(t)\left\{1+O\left(t^{-1}\right)\right\}, \quad t \rightarrow \infty
$$

where

$$
C_{k}=\left\{\prod_{s \neq k}\left(\lambda_{k}-\lambda_{s}\right)\right\}^{-1} .
$$

We also know from Liouville's formula $[4, \S 7]$ that $\widehat{W}(t)$ is nonvanishing when $|t|>\widehat{a}$. Accordingly, there is a finite constant $\widehat{C}_{k}$ such that

$$
\left|\widehat{W}_{k}(t) / \widehat{W}(t)\right| \leq \widehat{C}_{k}\left|e^{-\lambda_{k} t} t^{-\mu_{k}}\right|, \quad|t| \geq b .
$$

For the integration paths $Q_{k}(z)$, we impose the following conditions:

(i) $Q_{k}(z)$ lies in the annulus $|t| \geq b$.

(ii) As $t$ moves along $Q_{k}(z)$ away from $z$,

$$
\operatorname{Re}\left\{\left(\lambda_{k}-\lambda_{j}\right) t+\left(\mu_{k}-\mu_{j}\right) \ln t\right\}, \quad k \neq j,
$$

is nondecreasing.

As a consequence of the second condition, we are assured that

$$
\left|e^{\left(\lambda_{j}-\lambda_{k}\right) t} t^{\mu_{j}-\mu_{k}}\right| \leq\left|e^{\left(\lambda_{j}-\lambda_{k}\right) z} z^{\mu_{j}-\mu_{k}}\right|, \quad t \in Q_{k}(z) .
$$

Let us write

$$
\nu_{j}=\max _{k \neq j}\left|\frac{\mu_{k}-\mu_{j}}{\lambda_{k}-\lambda_{j}}\right|
$$

and assume that Condition (i) is satisfied. Then by following the analysis of $[15, \S 7]$, the roles of $t$ and $k$ in that reference now being played by $e^{i \theta_{j k}} t$ and $-\left(\mu_{k}-\mu_{j}\right) /\left\{2\left|\lambda_{k}-\lambda_{j}\right|\right\}$, respectively, we see that Condition (ii) is certainly satisfied if we take $b$ large enough to ensure that $b \geq \nu_{j} \sqrt{2}$ and also require that the angle between the direction of $Q_{k}(z)$, as $t$ moves along $Q_{k}(z)$ away from $z$, and the direction ph $t=-\theta_{j k}$ lies in the interval $\left[-\frac{1}{2} \pi+\kappa_{j}, \frac{1}{2} \pi-\kappa_{j}\right]$ where

$$
\kappa_{j} \equiv \sin ^{-1}\left(\nu_{j} / b\right) \in\left[0, \frac{1}{4} \pi\right] \text {. }
$$

The actual paths $Q_{k}(z)$ that we use depend on the value of $\beta_{j}-\alpha_{j}$ and the value of $\theta \equiv \mathrm{ph} z$. We shall need to distinguish between three main cases as follows.

Case I. $\beta_{j}-\alpha_{j} \in\left[0, \pi-2 \kappa_{j}\right], \quad \theta \in\left[-\frac{1}{2} \pi-\alpha_{j}+\kappa_{j}, \frac{1}{2} \pi-\beta_{j}-\kappa_{j}\right]$.

Case II. $\beta_{j}-\alpha_{j} \in\left[0, \pi-2 \kappa_{j}\right], \quad \theta \in\left(-\frac{3}{2} \pi-\alpha_{j}+\kappa_{j},-\frac{1}{2} \pi-\alpha_{j}+\kappa_{j}\right] \cup$ $\left[\frac{1}{2} \pi-\beta_{j}-\kappa_{j}, \frac{3}{2} \pi-\beta_{j}-\kappa_{j}\right)$.

Case III. $\beta_{j}-\alpha_{j} \in\left[\pi-2 \kappa_{j}, 2 \pi-4 \kappa_{j}\right], \quad \theta \in\left(-\frac{3}{2} \pi-\alpha_{j}+\kappa_{j}, \frac{3}{2} \pi-\beta_{j}-\kappa_{j}\right)$.

It will be observed that in the case $n=2$, we have $\alpha_{j}=\beta_{j}$, hence Case III does not arise. 


\section{Solution of the integral equation in Cases I and II}

Case I is depicted in Figure 7.1. In this and subsequent diagrams, each ray from the origin is labelled with its angular direction and $\theta=\mathrm{ph} z$.

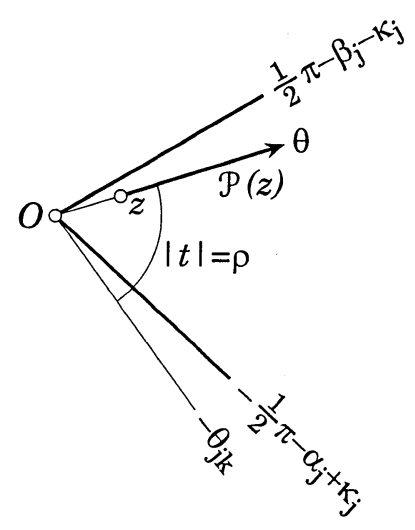

FiguRE 7.1. $t$-plane. $0 \leq \beta_{j}-\alpha_{j} \leq \pi-2 \kappa_{j}$ and $-\frac{1}{2} \pi-\alpha_{j}+\kappa_{j} \leq$ $\theta \leq \frac{1}{2} \pi-\beta_{j}-\kappa_{j}$.

We first show that if, for all $k$,

$$
S>\operatorname{Re}\left(\mu_{j}-\mu_{k}\right)
$$

then in (5.14) and (5.18), we can deform $Q_{k}(z)$ into the outward ray $\mathcal{P}(z)$ from $z$, that is,

$$
\mathcal{P}(z)=\{t: \operatorname{ph}(t-z)=\theta\}
$$

For large $|t|$, we have

$$
\left\{\widehat{W}_{k}(t) / \widehat{W}(t)\right\} e^{\lambda_{j} t} t^{\mu_{j}} T_{S j}(t)=O\left\{e^{-\left(\lambda_{k}-\lambda_{j}\right) t} t^{\mu_{j}-\mu_{k}-S-1}\right\} ;
$$

compare (6.1) and (6.7). Consider the circular arc $|t|=\rho$ running from ph $t=-\theta_{j k}$ to ph $t=\theta$. Suppose first that $k \neq j$. Then on this arc, ph $\left\{\left(\lambda_{k}-\lambda_{j}\right) t\right\} \equiv \theta_{j k}+\operatorname{ph} t$ runs from 0 to $\theta_{j k}+\theta$. Now

$$
\theta_{j k}+\theta \in\left[\theta_{j k}-\frac{1}{2} \pi-\alpha_{j}+\kappa_{j}, \theta_{j k}+\frac{1}{2} \pi-\beta_{j}-\kappa_{j}\right] \subseteq\left[-\frac{1}{2} \pi+\kappa_{j}, \frac{1}{2} \pi-\kappa_{j}\right]
$$

since $\alpha_{j} \leq \theta_{j k} \leq \beta_{j}$. Hence, $\left|e^{-\left(\lambda_{k}-\lambda_{j}\right) t}\right| \leq 1$. Obviously the last inequality continues to apply when $k=j$. Hence, from (7.1), the integrals around this arc of the integrands on the right-hand sides of (5.14) and (5.18) vanish as $\rho \rightarrow \infty$.

We next observe that as a consequence of (7.3), Conditions (i) and (ii) of $\S 6$ are satisfied by the choice $Q_{k}(z)=\mathcal{P}(z)$ as long as $|z| \geq b$. Hence, on substituting into (5.14) and (5.18) by means of (6.1), (6.3), (6.5), (6.9) and then applying (6.10), we obtain

$$
\left|h_{0}^{(\ell)}(z)\right| \leq B_{S j} D_{\ell}\left|e^{\lambda_{j} z} z^{\mu_{j}}\right| \mathcal{V}_{\mathcal{P}(z)}\left(t^{-S}\right)
$$


and

$$
\left|L_{2}\left\{h_{0}(z)\right\}\right| \leq B_{S j} G\left|e^{\lambda_{j} z} z^{\mu_{j}-2}\right| \mathcal{V}_{\mathcal{P}(z)}\left(t^{-S}\right)
$$

where

$$
D_{\ell}=\sum_{k=1}^{n} \widehat{C}_{k} D_{k \ell}, \quad G=\sum_{k=1}^{n} \widehat{C}_{k} G_{k}
$$

and $\mathcal{V}$ denotes the variational operator [16, Chapter $1, \S 11]$.

Next, consider (5.19) with $s=1$. We may substitute by means of (7.5). Since

$$
\nu_{\mathcal{P}(z)}\left(t^{-S}\right)=|z|^{-S}
$$

$[16$, Chapter $6, \S 13]$, it follows by analysis similar to that used at the beginning of this section that we again may take $Q_{k}(z)=\mathcal{P}(z)$. Then substituting by means of $(6.5)$ and (6.9), applying (6.10), and majorizing $\mathcal{V}_{\mathcal{P}(t)}\left(t^{-S}\right)$ by $\mathcal{V}_{\mathcal{P}(z)}\left(t^{-S}\right)$, we conclude that

$$
\left|L_{2}\left\{h_{1}(z)\right\}\right| \leq B_{S j} G^{2}\left|e^{\lambda_{j} z} z^{\mu_{j}-2}\right| \mathcal{V}_{\mathcal{P}(z)}\left(t^{-1}\right) \mathcal{V}_{\mathcal{P}(z)}\left(t^{-S}\right) .
$$

This process may be continued. On the next step, for example, we use

$$
\int_{\mathcal{P}(z)} \mathcal{V}_{\mathcal{P}(t)}\left(t^{-1}\right)\left|t^{-2} d t\right| \leq\left\{\mathcal{V}_{\mathcal{P}(z)}\left(t^{-1}\right)\right\}^{2} / 2
$$

By induction, we may verify that

$$
\left|L_{2}\left\{h_{s}(z)\right\}\right| \leq B_{S j}\left(G^{s+1} / s !\right)\left|e^{\lambda_{j} z} z^{\mu_{j}-2}\right|\left\{\mathcal{V}_{\mathcal{P}(z)}\left(t^{-1}\right)\right\}^{s} \mathcal{V}_{\mathcal{P}(z)}\left(t^{-S}\right)
$$

for $s=0,1, \ldots$ Then from (5.15), (6.3), (6.9), and (7.4), we derive

$$
\left|h_{s}^{(\ell)}(z)\right| \leq B_{S j} D_{\ell}\left(G^{s} / s !\right)\left|e^{\lambda_{j} z} z^{\mu_{j}}\right|\left\{\mathcal{V}_{\mathcal{P}(z)}\left(t^{-1}\right)\right\}^{s} \mathcal{V}_{\mathcal{P}(z)}\left(t^{-S}\right),
$$

$s \geq 0, \ell=0,1, \ldots, n-1$.

As a consequence, the series (5.10) converges uniformly on compact sets in the annular sector

$$
\left\{z:|z| \geq b \text { and } \theta \in\left[-\frac{1}{2} \pi-\alpha_{j}+\kappa_{j}, \frac{1}{2} \pi-\beta_{j}-\kappa_{j}\right]\right\} .
$$

Its sum is therefore analytic within, and continuous on, this sector. That $R_{S j}(z)$, defined in this way, satisfies (5.4) may be verified with the aid of (5.14)-(5.17). Then the function $w(z)$ defined by (5.3) is a solution of (1.1). Moreover, as a consequence of $(7.9)$, we have

$$
\left|\left(\frac{d}{d z}\right)^{\ell} R_{S j}(z)\right| \leq B_{S j} D_{\ell}\left|e^{\lambda_{j} z} z^{\mu_{j}}\right| \mathcal{V}_{\mathcal{P}(z)}\left(t^{-S}\right) \exp \left\{G \mathcal{V}_{\mathcal{P}(z)}\left(t^{-1}\right)\right\},
$$

$\ell=0,1, \ldots, n-1$.

We now consider Case II and suppose first that $\theta \in\left[\frac{1}{2} \pi-\beta_{j}-\kappa_{j}, \frac{3}{2} \pi-\beta_{j}-\kappa_{j}\right)$; see Figures 7.2 and 7.3.

Unlike Case I, the paths $\mathcal{Q}_{k}(z)$ cannot be deformed into the outward ray $\mathcal{P}(z)$ from $z$ for all values of $k$. (This is true, for example, of the value of $k$ for which $\theta_{j k}=\beta_{j}$.) However, as long as (7.1) continues to hold, we may for each $k$ deform $Q_{k}(z)$ into $\mathcal{P}_{1}(z)$, where $\mathcal{P}_{1}(z)$ is the half-line emerging from $z$ parallel to the right-hand boundary of the given sector, that is,

$$
\mathcal{P}_{1}(z)=\left\{t: \operatorname{ph}(t-z)=\frac{1}{2} \pi-\beta_{j}-\kappa_{j}\right\} .
$$




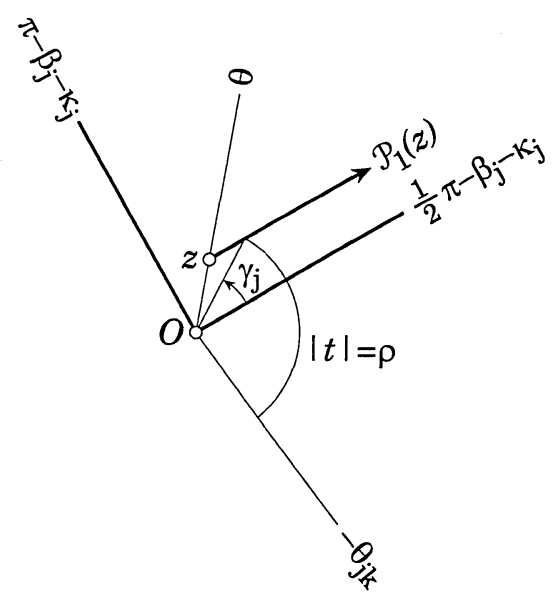

FIgURE 7.2. $t$-plane. $0 \leq \beta_{j}-\alpha_{j} \leq \pi-2 \kappa_{j}$ and $\frac{1}{2} \pi-\beta_{j}-\kappa_{j} \leq \theta \leq$ $\pi-\beta_{j}-\kappa_{j}$.

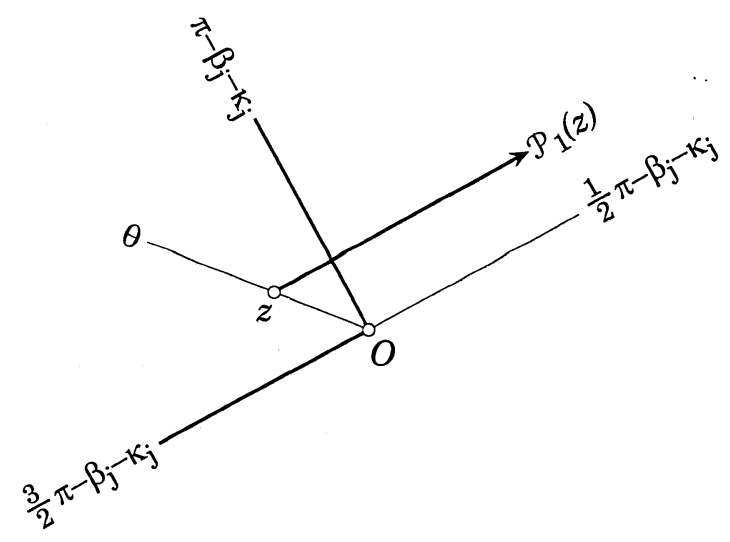

FIGURE 7.3. $t$-plane. $0 \leq \beta_{j}-\alpha_{j} \leq \pi-2 \kappa_{j}$ and $\pi-\beta_{j}-\kappa_{j} \leq \theta<$ $\frac{3}{2} \pi-\beta_{j}-\kappa_{j}$.

This may be proved in a similar way to Case I. On the part of the circular arc $|t|=\rho$ between the ray ph $t=-\theta_{j k}$ and $\mathcal{P}_{1}(z)$, ph $t$ runs from $-\theta_{j k}$ to $\frac{1}{2} \pi-\beta_{j}-\kappa_{j}+\gamma_{j}$ where $0 \leq \gamma_{j} \leq O(1 / \rho)$ as $\rho \rightarrow \infty$. Hence, ph $\left\{\left(\lambda_{k}-\lambda_{j}\right) t\right\}$ runs from 0 to

$$
\begin{aligned}
\theta_{j k}+\frac{1}{2} \pi-\beta_{j}-\kappa_{j}+\gamma_{j} & \in\left[\frac{1}{2} \pi-\left(\beta_{j}-\alpha_{j}\right)-\kappa_{j}+\gamma_{j}, \frac{1}{2} \pi-\kappa_{j}+\gamma_{j}\right] \\
& \subseteq\left[-\frac{1}{2} \pi+\kappa_{j}+\gamma_{j}, \frac{1}{2} \pi-\kappa_{j}+\gamma_{j}\right] \\
& \subseteq\left[-\frac{1}{2} \pi, \frac{1}{2} \pi+\gamma_{j}\right] .
\end{aligned}
$$


Therefore,

$$
-\operatorname{Re}\left\{\left(\lambda_{k}-\lambda_{j}\right) t\right\} \leq\left|\lambda_{k}-\lambda_{j}\right| \rho \sin \gamma_{j}=O(1) ;
$$

accordingly, $\left|e^{-\left(\lambda_{k}-\lambda_{j}\right) t}\right|$ is uniformly bounded on the arc as $\rho \rightarrow \infty$.

Next, we observe that Condition (i) of $\S 6$ is satisfied if

$$
|z| \geq b \text { when } \frac{1}{2} \pi-\beta_{j}-\kappa_{j} \leq \theta \leq \pi-\beta_{j}-\kappa_{j}
$$

(Figure 7.2), or

$$
|z| \geq b \operatorname{cosec}\left(\frac{3}{2} \pi-\beta_{j}-\kappa_{j}-\theta\right) \text { when } \pi-\beta_{j}-\kappa_{j} \leq \theta<\frac{3}{2} \pi-\beta_{j}-\kappa_{j}
$$

(Figure 7.3). We also observe that the angle from the ray ph $t=-\theta_{j k}$ to the direction of $\mathcal{P}_{1}(z)$ is

$$
\frac{1}{2} \pi-\beta_{j}-\kappa_{j}+\theta_{j k} \in\left[\frac{1}{2} \pi-\left(\beta_{j}-\alpha_{j}\right)-\kappa_{j}, \frac{1}{2} \pi-\kappa_{j}\right] \subseteq\left[-\frac{1}{2} \pi+\kappa_{j}, \frac{1}{2} \pi-\kappa_{j}\right] .
$$

Accordingly, Condition (ii) is satisfied.

The rest of the analysis is similar to that of Case I, with $\mathcal{P}(z)$ replaced everywhere by $\mathcal{P}_{1}(z)$. In place of $(7.10)$, we have

$$
\left|\left(\frac{d}{d z}\right)^{\ell} R_{S j}(z)\right| \leq B_{S j} D_{\ell}\left|e^{\lambda_{j} z} z^{\mu_{j}}\right| \mathcal{V}_{\mathcal{P}_{1}(z)}\left(t^{-S}\right) \exp \left\{G \mathcal{V}_{\mathcal{P}_{1}(z)}\left(t^{-1}\right)\right\},
$$

$\ell=0,1, \ldots, n-1$.

Lastly, we consider Case II when $\theta \in\left(-\frac{3}{2} \pi-\alpha_{j}+\kappa_{j},-\frac{1}{2} \pi-\alpha_{j}+\kappa_{j}\right]$. The diagrams of the $t$-plane for this case are similar to Figures 7.2 and 7.3 and are omitted.

We now deform $\mathcal{Q}_{k}(z)$ into the half-line $\mathcal{P}_{2}(z)$ emerging from $z$ parallel to the lefthand boundary of the given sector:

$$
\mathcal{P}_{2}(z)=\left\{t: \operatorname{ph}(t-z)=-\frac{1}{2} \pi-\alpha_{j}+\kappa_{j}\right\} .
$$

The final result is given by

$$
\left|\left(\frac{d}{d z}\right)^{\ell} R_{S j}(z)\right| \leq B_{S_{j}} D_{\ell}\left|e^{\lambda_{j} z} z^{\mu_{j}}\right| \mathcal{V}_{\mathcal{P}_{2}(z)}\left(t^{-S}\right) \exp \left\{G \mathcal{V}_{\mathcal{P}_{2}(z)}\left(t^{-1}\right)\right\}
$$

$\ell=0,1, \ldots, n-1$. This is valid when (7.1) holds and

$$
|z| \geq b \quad \text { when }-\pi-\alpha_{j}+\kappa_{j} \leq \theta \leq-\frac{1}{2} \pi-\alpha_{j}+\kappa_{j},
$$

or

$$
|z| \geq b \operatorname{cosec}\left(\theta+\frac{3}{2} \pi+\alpha_{j}-\kappa_{j}\right) \quad \text { when }-\frac{3}{2} \pi-\alpha_{j}+\kappa_{j}<\theta \leq-\pi-\alpha_{j}+\kappa_{j} .
$$

Remark. The analysis in this section could be shortened by appealing to general theorems for solving singular Volterra integral equations given in [16, Chapter $6, \S 10]$. We did not adopt this course since much of the analysis is relevant to the more difficult situation in which $\beta_{j}-\alpha_{j}$ exceeds $\pi-2 \kappa_{j}$. This case is treated in the next section, and the cited theorems no longer apply. 


\section{Solution of the integral equation in Case III}

When $\beta_{j}-\alpha_{j}$ exceeds $\pi-2 \kappa_{j}$, it is no longer possible to deform all the paths $Q_{k}(z)$ into a single half-line from $z$ to the point at infinity. Instead, we employ two such half-lines, in fact, the paths $\mathcal{P}_{1}(z)$ and $\mathcal{P}_{2}(z)$ defined by (7.11) and (7.13).

Figures 8.1 and 8.2 illustrate the paths $\mathcal{P}_{1}(z)$ and $\mathcal{P}_{2}(z)$ when $\theta$ lies in the subinterval $\left[-\pi-\alpha_{j}+\kappa_{j}, \pi-\beta_{j}-\kappa_{j}\right]$. Figure 8.1 pertains to the range $\beta_{j}-\alpha_{j} \in\left[\pi-2 \kappa_{j}, \frac{3}{2} \pi-2 \kappa_{j}\right]$ for which the angle from $\mathcal{P}_{1}(z)$ to $\mathcal{P}_{2}(z)$ is in the interval $\left[0, \frac{1}{2} \pi\right]$, and Figure 8.2 pertains to the range $\beta_{j}-\alpha_{j} \in\left[\frac{3}{2} \pi-2 \kappa_{j}, 2 \pi-4 \kappa_{j}\right]$ for which the angle from $\mathcal{P}_{1}(z)$ to $\mathcal{P}_{2}(z)$

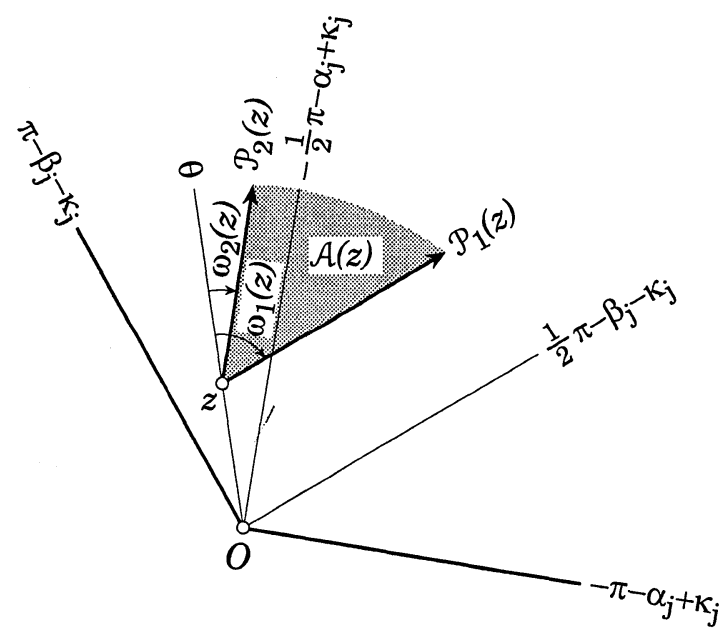

FIGURE 8.1. $t$-plane. $\pi-2 \kappa_{j} \leq \beta_{j}-\alpha_{j} \leq \frac{3}{2} \pi-2 \kappa_{j}$ and $-\pi-\alpha_{j}+\kappa_{j} \leq$ $\theta \leq \pi-\beta_{j}-\kappa_{j}$.

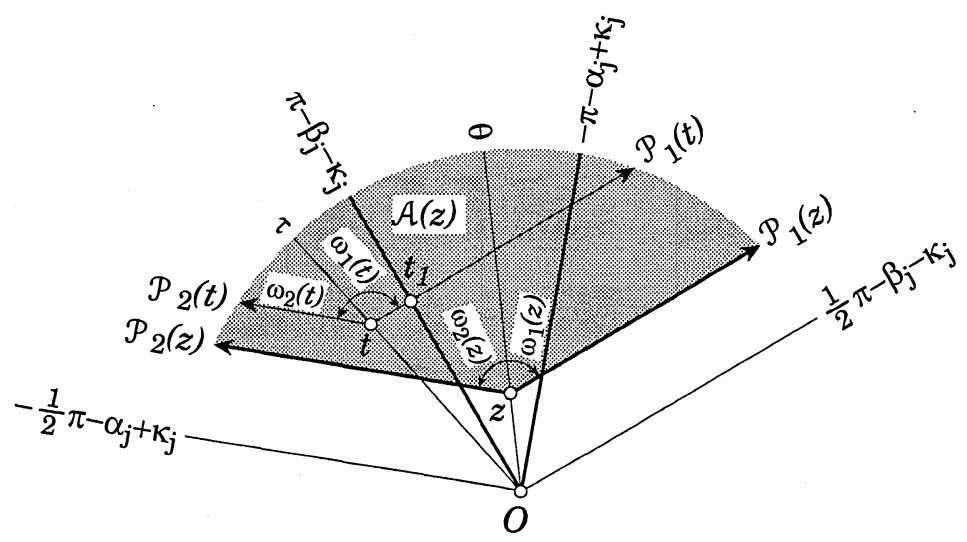

FigURE 8.2. $t$-plane. $\frac{3}{2} \pi-2 \kappa_{j} \leq \beta_{j}-\alpha_{j} \leq 2 \pi-4 \kappa_{j}$ and $-\pi-\alpha_{j}+$ $\kappa_{j} \leq \theta \leq \pi-\beta_{j}-\kappa_{j}$. 
is in $\left[\frac{1}{2} \pi, \pi-2 \kappa_{j}\right]$. In a similar manner, Figures 8.3 and 8.4 depict $\mathcal{P}_{1}(z)$ and $\mathcal{P}_{2}(z)$ when $\theta \in\left[\pi-\beta_{j}-\kappa_{j}, \frac{3}{2} \pi-\beta_{j}-\kappa_{j}\right)$. The corresponding diagrams for the remaining range $\theta \in\left(-\frac{3}{2} \pi-\alpha_{j}+\kappa_{j},-\pi-\alpha_{j}+\kappa_{j}\right]$ are similar to Figures 8.3 and 8.4 and are omitted.

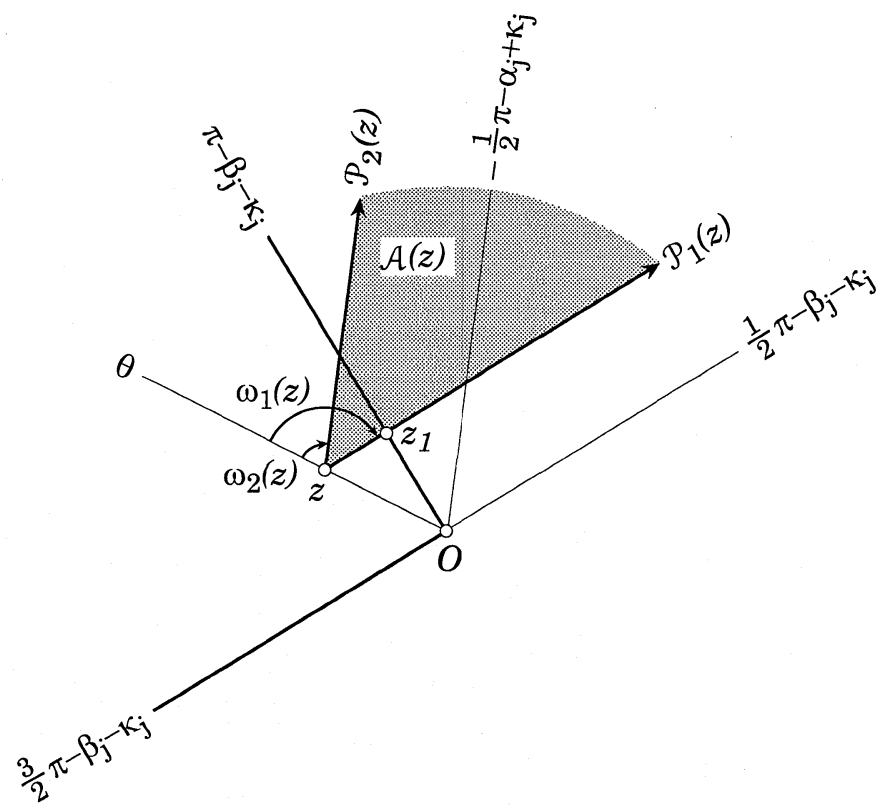

FIGURE 8.3. $t$-plane. $\pi-2 \kappa_{j} \leq \beta_{j}-\alpha_{j} \leq \frac{3}{2} \pi-2 \kappa_{j}$ and $\pi-\beta_{j}-\kappa_{j} \leq$ $\theta<\frac{3}{2} \pi-\beta_{j}-\kappa_{j}$.

In equations (5.14)-(5.19), we take

$$
Q_{k}(z)= \begin{cases}\mathcal{P}_{1}(z) & \text { when } \theta_{j k} \in\left[\frac{1}{2} \alpha_{j}+\frac{1}{2} \beta_{j}, \beta_{j}\right] \\ \mathcal{P}_{2}(z) & \text { when } \theta_{j k} \in\left[\alpha_{j}, \frac{1}{2} \alpha_{j}+\frac{1}{2} \beta_{j}\right] .\end{cases}
$$

(If $\theta_{j k}=\frac{1}{2} \alpha_{j}+\frac{1}{2} \beta_{j}$, as happens when $k=j$, then we may take $Q_{k}(z)$ to be either $\mathcal{P}_{1}(z)$ or $\left.\mathcal{P}_{2}(z)\right)$. The justification of these deformations of the integration paths is similar to that given in $\S 7$.

In order to satisfy Condition (i) of $\S 6$, we need to examine the angles $\omega_{1}(z)$ and $\omega_{2}(z)$, say, from ph $t=\theta$ to $\mathcal{P}_{1}(z)$ and $\mathcal{P}_{2}(z)$, respectively. These angles are indicated on Figures 8.1-8.4 and are given by

$$
\omega_{1}(z)=\frac{1}{2} \pi-\beta_{j}-\kappa_{j}-\theta, \quad \omega_{2}(z)=-\frac{1}{2} \pi-\alpha_{j}+\kappa_{j}-\theta .
$$

Suppose first that $\theta \in\left[-\pi-\alpha_{j}+\kappa_{j}, \pi-\beta_{j}-\kappa_{j}\right]$ (Figures 8.1 and 8.2). Then

$$
\omega_{1}(z) \in\left[-\frac{1}{2} \pi, \frac{3}{2} \pi-\left(\beta_{j}-\alpha_{j}\right)-2 \kappa_{j}\right] \subseteq\left[-\frac{1}{2} \pi, 0\right],
$$

and

$$
\omega_{2}(z) \in\left[-\frac{3}{2} \pi+\beta_{j}-\alpha_{j}+2 \kappa_{j}, \frac{1}{2} \pi\right] \subseteq\left[0, \frac{1}{2} \pi\right]
$$




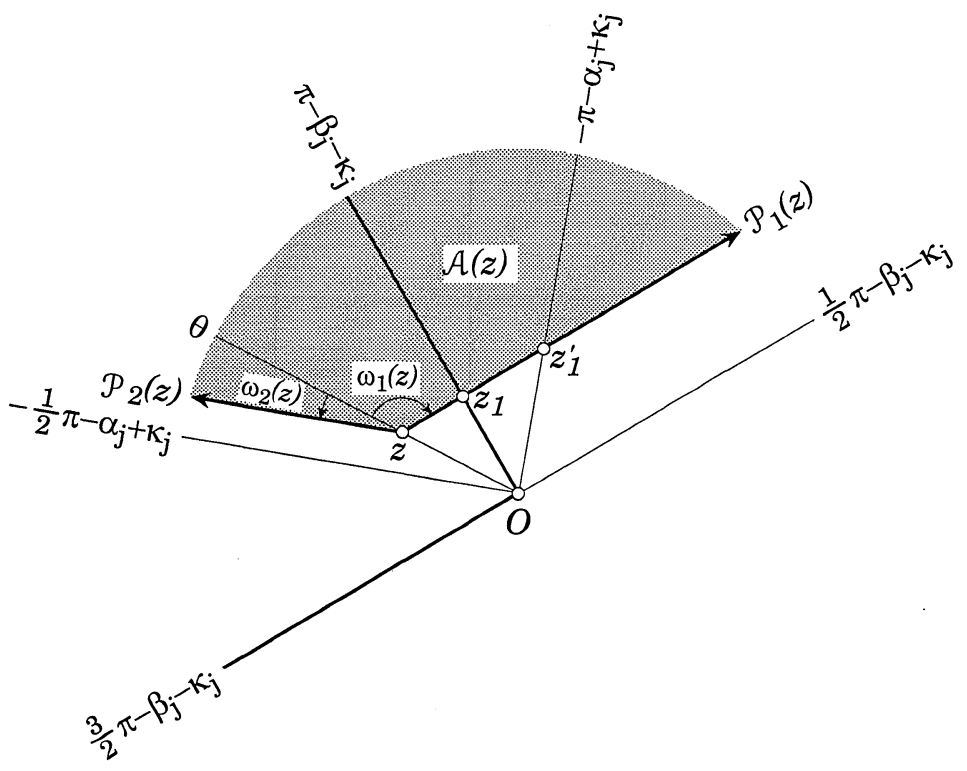

FIGURE 8.4. $t$-plane. $\frac{3}{2} \pi-2 \kappa_{j} \leq \beta_{j}-\alpha_{j} \leq 2 \pi-4 \kappa_{j}$ and $\pi-\beta_{j}-\kappa_{j} \leq$ $\theta<\frac{3}{2} \pi-\beta_{j}-\kappa_{j}$.

Accordingly, Condition (i) is satisfied when $|z| \geq b$.

Next, suppose that $\theta \in\left[\pi-\beta_{j}-\kappa_{j}, \frac{3}{2} \pi-\beta_{j}-\kappa_{j}\right.$ ) (Figures 8.3 and 8.4.), then

$$
\omega_{1}(z) \in\left(-\pi,-\frac{1}{2} \pi\right] \text {. }
$$

Also,

$$
\begin{aligned}
-\omega_{1}(z)-\omega_{2}(z)=2 \theta+\alpha_{j}+\beta_{j} & \in\left[2 \pi-\left(\beta_{j}-\alpha_{j}\right)-2 \kappa_{j}, 3 \pi-\left(\beta_{j}-\alpha_{j}\right)-2 \kappa_{j}\right) \\
& \subseteq\left[2 \kappa_{j}, 2 \pi\right),
\end{aligned}
$$

and

$$
-\omega_{1}(z)+\omega_{2}(z)=-\pi+\beta_{j}-\alpha_{j}+2 \kappa_{j} \in\left[0, \pi-2 \kappa_{j}\right] .
$$

These three equations show that $\left|\omega_{2}(z)\right| \leq\left|\omega_{1}(z)\right|<\pi$, and hence that Condition (i) is satisfied when $|z| \geq b \operatorname{cosec}\left\{\pi+\omega_{1}(z)\right\}$, that is, $|z| \geq b \operatorname{cosec}\left(\frac{3}{2} \pi-\beta_{j}-\kappa_{j}-\theta\right)$.

On carrying out a similar analysis for the remaining range $\theta \in\left(-\frac{3}{2} \pi-\alpha_{j}+\kappa_{j}\right.$, $-\pi-\alpha_{j}+\kappa_{j}$ ] and summarizing the results, we see that Condition (i) of $\S 6$ is satisfied when

$$
\begin{gathered}
|z| \geq b, \quad-\pi-\alpha_{j}+\kappa_{j} \leq \theta \leq \pi-\beta_{j}-\kappa_{j} \\
|z| \geq b \operatorname{cosec}\left(\frac{3}{2} \pi-\beta_{j}-\kappa_{j}-\theta\right), \quad \pi-\beta_{j}-\kappa_{j} \leq \theta<\frac{3}{2} \pi-\beta_{j}-\kappa_{j}, \\
|z| \geq b \operatorname{cosec}\left(\theta+\frac{3}{2} \pi+\alpha_{j}-\kappa_{j}\right), \quad-\frac{3}{2} \pi-\alpha_{j}+\kappa_{j}<\theta \leq-\pi-\alpha_{j}+\kappa_{j} .
\end{gathered}
$$

That Condition (ii) of $\S 6$ is satisfied in these circumstances again follows as in $\S 7$. 
With the choice (8.1), we find that on substituting into (5.14) by means of (6.1), (6.3), and (6.9), and applying (6.10), we have

$$
\left|h_{0}^{(\ell)}(z)\right| \leq B_{S j}\left|e^{\lambda_{j} z} z^{\mu_{j}}\right| U_{S j \ell}(z) .
$$

Here

$$
U_{S j \ell}(z)=\widehat{D}_{1 \ell} \mathcal{V}_{\mathcal{P}_{1}(z)}\left(t^{-S}\right)+\widehat{D}_{2 \ell} \mathcal{V}_{\mathcal{P}_{2}(z)}\left(t^{-S}\right)
$$

with

$$
\widehat{D}_{1 \ell}=\sum_{1} \widehat{C}_{k} D_{k \ell}, \quad \widehat{D}_{2 \ell}=\sum_{2} \widehat{C}_{k} D_{k \ell}
$$

where $\sum_{1}$ denotes the sum over those values of $k$ for which $Q_{k}(z)=\mathcal{P}_{1}(z)$, and $\sum_{2}$ denotes the sum over those values of $k$ for which $Q_{k}(z)=\mathcal{P}_{2}(z)$. Similarly, from (5.18), we derive

$$
\left|L_{2}\left\{h_{0}(z)\right\}\right| \leq B_{S j}\left|e^{\lambda_{j} z} z^{\mu_{j}-2}\right| V_{S j}(z)
$$

where

$$
V_{S j}(z)=\widehat{G}_{1} \mathcal{V}_{\mathcal{P}_{1}(z)}\left(t^{-S}\right)+\widehat{G}_{2} \mathcal{V}_{\mathcal{P}_{2}(z)}\left(t^{-S}\right)
$$

and

$$
\widehat{G}_{1}=\sum_{1} \widehat{C}_{k} G_{k}, \quad \widehat{G}_{2}=\sum_{2} \widehat{C}_{k} G_{k}
$$

Before we can continue to the next step, we need to show that (8.9) and (8.10) remain valid when $z$ is replaced by $t$ with $t \in \mathcal{P}_{1}(z) \cup \mathcal{P}_{2}(z)$, that is, $t$ lies on the boundary of the closed sector $\mathcal{A}(z)$ obtained by rotating $\mathcal{P}_{1}(z)$ around $z$ in the positive sense to $\mathcal{P}_{2}(z) . \mathcal{A}(z)$ is indicated by the shading in Figures 8.1-8.4. Since the angle $-\pi+\beta_{j}-\alpha_{j}+2 \kappa_{j}$ of $\mathcal{A}(z)$ is less than $\pi$, both $\mathcal{P}_{1}(t)$ and $\mathcal{P}_{2}(t)$ lie in $\mathcal{A}(z)$. Hence, if (8.3)-(8.5) apply, then $\mathcal{P}_{1}(t)$ and $\mathcal{P}_{2}(t)$ each satisfy Conditions (i) and (ii) of $\S 6$; as a consequence $z$ may be replaced by $t$ in (8.9) and (8.10). Then from (5.19) with $s=1$, we derive

$$
\begin{aligned}
\left|L_{2}\left\{h_{1}(z)\right\}\right| & \leq B_{S j}\left|e^{\lambda_{j} z} z^{\mu_{j}-2}\right| \sum_{k=1}^{n} \widehat{C}_{k} G_{k} \int_{Q_{k}(z)} V_{S j}(t)\left|t^{-2} d t\right| \\
& \leq B_{S j}\left|e^{\lambda_{j} z} z^{\mu_{j}-2}\right| \widehat{V}_{S j}(z) V_{1 j}(z)
\end{aligned}
$$

where

$$
\widehat{V}_{S j}(z)=\sup _{t \in \mathcal{A}(z)} V_{S j}(t) .
$$

On the following step, we have

$$
\begin{aligned}
\left|L_{2}\left\{h_{2}(z)\right\}\right| & \leq B_{S j}\left|e^{\lambda_{j} z} z^{\mu_{j}-2}\right| \sum_{k=1}^{n} \widehat{C}_{k} G_{k} \int_{Q_{k}(z)} \widehat{V}_{S j}(t) V_{1 j}(t)\left|t^{-2} d t\right| \\
& \leq B_{S j}\left|e^{\lambda_{j} z} z^{\mu_{j}-2}\right| \widehat{V}_{S j}(z) \widehat{V}_{1 j}(z) V_{1 j}(z) .
\end{aligned}
$$

(Here we have used the fact that $\mathcal{A}(t) \subseteq \mathcal{A}(z)$ when $t \in \mathcal{A}(z)$.)

The cycle of steps may be continued, and we may verify by induction that

$$
\left|L_{2}\left\{h_{s}(z)\right\}\right| \leq B_{S j}\left|e^{\lambda_{j} z} z^{\mu_{j}-2}\right| \widehat{V}_{S j}(z)\left\{\widehat{V}_{1 j}(z)\right\}^{s-1} V_{1 j}(z) .
$$


As a consequence,

$$
\left|L_{2}\left\{h_{s}(z)\right\}\right| \leq B_{S j}\left|e^{\lambda_{j} z} z^{\mu_{j}-2}\right| \widehat{V}_{S j}(z)\left\{\widehat{V}_{1 j}(z)\right\}^{s} ;
$$

this result also holds for $s=0$ by virtue of (8.9). Then from (5.15), we obtain

$$
\left|h_{s}^{(\ell)}(z)\right| \leq B_{S j}\left|e^{\lambda_{j} z} z^{\mu_{j}}\right| \widehat{V}_{S j}(z)\left\{\widehat{V}_{1 j}(z)\right\}^{s-1} U_{1 j \ell}(z), \quad s \geq 1 .
$$

Summation of (8.6) and (8.15) yields the desired bound:

$$
\left|\left(\frac{d}{d z}\right)^{\ell} R_{S j}(z)\right|=\left|\sum_{s=0}^{\infty} h_{s}^{(\ell)}(z)\right| \leq B_{S j}\left|e^{\lambda_{j} z} z^{\mu_{j}}\right|\left\{U_{S j \ell}(z)+\frac{\widehat{V}_{S j}(z) U_{1 j \ell}(z)}{1-\widehat{V}_{1 j}(z)}\right\},
$$

provided that $\ell=0,1, \ldots, n-1$ and $\widehat{V}_{1 j}(z)<1$. The other conditions needed for the validity of this result are (7.1) and (8.3)-(8.5).

\section{Evaluation and simplification of the error bounds}

In this section, we digress on the problem of evaluating the bounds that were constructed in $\S \S 7$ and 8 for the remainder term $R_{S j}(z)$ and its derivatives. Besides the intrinsic interest of these bounds for computational purposes, the results we derive here ease the understanding of the completion of the proof of the theorems of $\S 3$ in the next section.

All of the bounds in $\S \S 7$ and 8 involve the variation of the function $t^{-S}$ along halflines in $\mathbb{C}, S$ being a positive integer. The general parametric equation of such a line $\mathcal{Q}(z)$ that does not intersect the origin can be written

$$
\mathcal{Q}(z)=\left\{t: t=z\left(1+v e^{i \sigma}\right), 0 \leq v<\infty\right\}
$$

where $z(\neq 0)$ is the endpoint of $\mathcal{Q}(z)$, and $\sigma \in(-\pi, \pi)$ is the angle of slope of $\mathcal{Q}(z)$ to the ray ph $t=\theta$; see Figures 9.1 and 9.2.

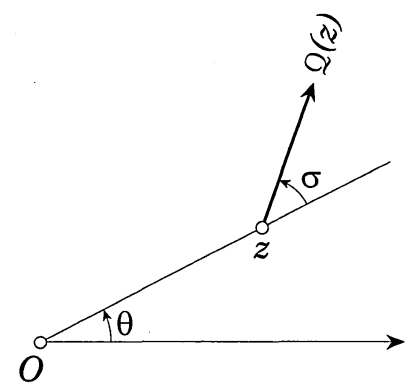

FiguRE 9.1. $Q(z)$ when $0 \leq|\sigma| \leq \frac{1}{2} \pi$.

By definition,

$$
\begin{aligned}
\nu_{Q(z)}\left(t^{-S}\right) & =S \int_{Q(z)}\left|\frac{d t}{t^{S+1}}\right|=\frac{S}{|z|^{S}} \int_{0}^{\infty} \frac{d v}{\left|1+v e^{i \sigma}\right|^{S+1}} \\
& =\frac{S}{|z|^{S}} \int_{0}^{\infty} \frac{d v}{\left(1+2 v \cos \sigma+v^{2}\right)^{(S+1) / 2}}
\end{aligned}
$$




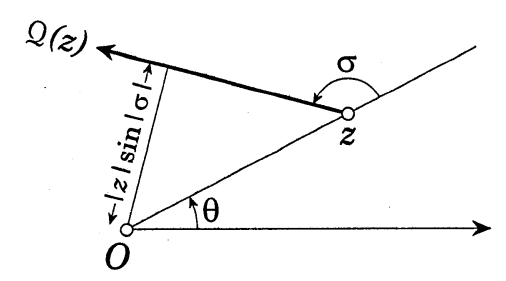

FiguRE 9.2. $Q(z)$ when $\frac{1}{2} \pi \leq|\sigma|<\pi$.

On taking a new integration variable $\psi$ defined by

$$
v+\cos \sigma=\sin \sigma \cot \psi
$$

we obtain

$$
\nu_{Q(z)}\left(t^{-S}\right)=I_{S}(\sigma)|z|^{-S}
$$

where

$$
I_{S}(\sigma)=\frac{S}{(\sin \sigma)^{S}} \int_{0}^{\sigma}(\sin \psi)^{S-1} d \psi
$$

For example,

$$
I_{1}(\sigma)=\frac{\sigma}{\sin \sigma}, \quad I_{2}(\sigma)=\frac{1}{\cos ^{2}\left(\frac{1}{2} \sigma\right)},
$$

and for higher values of $S$, we have the recurrence relation

$$
S \sin ^{2} \sigma I_{S+1}(\sigma)=(S+1) I_{S-1}(\sigma)-(S+1) \cos \sigma, \quad S \geq 2 .
$$

These identities can be used to generate $I_{S}(\sigma)$ analytically. For numerical purposes, however, there is some obvious instability lurking in the forward application of (9.6) when $|\sigma|$ is small.

We also observe from (9.2) that when $\sigma=0$,

$$
v_{Q(z)}\left(t^{-S}\right)=|z|^{-S}
$$

furthermore, $\mathcal{V}_{\mathcal{Q}(z)}\left(t^{-S}\right)$ is an increasing function of $|\sigma|$. The latter observation enables us to construct simple, and not too unrealistic, bounds for $\mathcal{V}_{\mathcal{Q}(z)}\left(t^{-S}\right)$. From the Beta function integral, we have

$$
I_{S}\left(\frac{1}{2} \pi\right)=\chi(S)
$$

where

$$
\chi(S)=\pi^{1 / 2} \Gamma\left(\frac{1}{2} S+1\right) / \Gamma\left(\frac{1}{2} S+\frac{1}{2}\right)
$$

Hence,

$$
\nu_{\mathcal{Q}(z)}\left(t^{-S}\right) \leq \chi(S)|z|^{-S}, \quad|\sigma| \leq \frac{1}{2} \pi .
$$

When $|\sigma| \geq \frac{1}{2} \pi$, the shortest distance from the origin to $Q(z)$ is $|z| \sin |\sigma|$; compare Figure 9.2. Accordingly,

$$
\mathcal{V}_{Q(z)}\left(t^{-S}\right)<2 \chi(S)|\operatorname{cosec} \sigma|^{S}|z|^{-S}, \quad \frac{1}{2} \pi \leq|\sigma|<\pi .
$$


This inequality also is given in [16, Chapter $6, \S 13]$, and this reference may be consulted for further information concerning the function $\chi(S)$.

We now may substitute straightforwardly by means of (9.7), (9.10), and (9.11) into the bounds for $(d / d z)^{\ell} R_{S j}(z)$ that were obtained in $\S \S 7$ and 8 . For brevity, we denote

$$
\left(\frac{d}{d z}\right)^{\ell} R_{S j}(z)=B_{S j} D_{\ell} e^{\lambda_{j} z} z^{\mu_{j}-S} R
$$

again for $\ell=0,1, \ldots, n-1$. Assuming that (7.1) continues to apply, we now state the final bounds for Cases I and II. In these results, the quantities $b$ and $\kappa_{j}$ are defined in $\S 6, G$ in $\S 7$, and $\chi(S)$ in the present section.

Case I. $\beta_{j}-\alpha_{j} \in\left[0, \pi-2 \kappa_{j}\right], \quad \theta \in\left[-\frac{1}{2} \pi-\alpha_{j}+\kappa_{j}, \frac{1}{2} \pi-\beta_{j}-\kappa_{j}\right]$.

$$
|R| \leq \exp \left(G|z|^{-1}\right), \quad|z| \geq b \text {. }
$$

Case IIa. $\beta_{j}-\alpha_{j} \in\left[0, \pi-2 \kappa_{j}\right], \quad \theta \in\left[-\pi-\alpha_{j}+\kappa_{j},-\frac{1}{2} \pi-\alpha_{j}+\kappa_{j}\right] \cup$ $\left[\frac{1}{2} \pi-\beta_{j}-\kappa_{j}, \pi-\beta_{j}-\kappa_{j}\right]$.

$$
|R| \leq \chi(S) \exp \left(\frac{1}{2} \pi G|z|^{-1}\right), \quad|z| \geq b .
$$

Case IIb. $\beta_{j}-\alpha_{j} \in\left[0, \pi-2 \kappa_{j}\right], \quad \theta \in\left(-\frac{3}{2} \pi-\alpha_{j}+\kappa_{j},-\pi-\alpha_{j}+\kappa_{j}\right]$.

$$
\begin{gathered}
|R| \leq 2 \chi(S) \operatorname{cosec}^{S}\left(\theta+\frac{3}{2} \pi+\alpha_{j}-\kappa_{j}\right) \exp \left\{\pi G \operatorname{cosec}\left(\theta+\frac{3}{2} \pi+\alpha_{j}-\kappa_{j}\right)|z|^{-1}\right\}, \\
|z| \geq b \operatorname{cosec}\left(\theta+\frac{3}{2} \pi+\alpha_{j}-\kappa_{j}\right) .
\end{gathered}
$$

Case IIc. $\beta_{j}-\alpha_{j} \in\left[0, \pi-2 \kappa_{j}\right], \quad \theta \in\left[\pi-\beta_{j}-\kappa_{j}, \frac{3}{2} \pi-\beta_{j}-\kappa_{j}\right)$.

$$
\begin{gathered}
|R| \leq 2 \chi(S) \operatorname{cosec}^{S}\left(\frac{3}{2} \pi-\beta_{j}-\kappa_{j}-\theta\right) \exp \left\{\pi G \operatorname{cosec}\left(\frac{3}{2} \pi-\beta_{j}-\kappa_{j}-\theta\right)|z|^{-1}\right\} \\
|z| \geq b \operatorname{cosec}\left(\frac{3}{2} \pi-\beta_{j}-\kappa_{j}-\theta\right) .
\end{gathered}
$$

In Case III, however, the analysis is less direct. We need to distinguish six subcases, as follows:

Case IIIa. $\beta_{j}-\alpha_{j} \in\left[\pi-2 \kappa_{j}, \frac{3}{2} \pi-2 \kappa_{j}\right], \quad \theta \in\left[-\pi-\alpha_{j}+\kappa_{j}, \pi-\beta_{j}-\kappa_{j}\right]$ (Figure 8.1).

Case IIIb. $\beta_{j}-\alpha_{j} \in\left[\frac{3}{2} \pi-2 \kappa_{j}, 2 \pi-4 \kappa_{j}\right], \quad \theta \in\left[-\pi-\alpha_{j}+\kappa_{j}, \pi-\beta_{j}-\kappa_{j}\right]$ (Figure 8.2).

Case IIIc. $\beta_{j}-\alpha_{j} \in\left[\pi-2 \kappa_{j}, \frac{3}{2} \pi-2 \kappa_{j}\right], \quad \theta \in\left(-\frac{3}{2} \pi-\alpha_{j}+\kappa_{j},-\pi-\alpha_{j}+\kappa_{j}\right]$ (not illustrated).

Case IIId. $\beta_{j}-\alpha_{j} \in\left[\frac{3}{2} \pi-2 \kappa_{j}, 2 \pi-4 \kappa_{j}\right], \quad \theta \in\left(-\frac{3}{2} \pi-\alpha_{j}+\kappa_{j},-\pi-\alpha_{j}+\kappa_{j}\right]$ (not illustrated).

Case IIIe. $\beta_{j}-\alpha_{j} \in\left[\pi-2 \kappa_{j}, \frac{3}{2} \pi-2 \kappa_{j}\right], \quad \theta \in\left[\pi-\beta_{j}-\kappa_{j}, \frac{3}{2} \pi-\beta_{j}-\kappa_{j}\right)$

(Figure 8.3). 
Case IIIf. $\beta_{j}-\alpha_{j} \in\left[\frac{3}{2} \pi-2 \kappa_{j}, 2 \pi-4 \kappa_{j}\right], \quad \theta \in\left[\pi-\beta_{j}-\kappa_{j}, \frac{3}{2} \pi-\beta_{j}-\kappa_{j}\right)$

(Figure 8.4).

Lemma 9.1. Assume $t \in \mathcal{A}(z)$ (as defined in $\S 8$ ). Then $\mathcal{V}_{\mathcal{P}_{1}(t)}\left(t^{-S}\right)$ and $\mathcal{V}_{\mathcal{P}_{2}(t)}\left(t^{-S}\right)$ are bounded by

$$
\begin{aligned}
& \chi(S)|z|^{-S} \text { in Case IIIa; } \quad 2 \chi(S)|z|^{-S} \text { in Case IIIb; } \\
& 2 \chi(S) \operatorname{cosec}^{S}\left(\theta+\frac{3}{2} \pi+\alpha_{j}-\kappa_{j}\right)|z|^{-S} \text { in Cases IIIc and IIId; } \\
& 2 \chi(S) \operatorname{cosec}^{S}\left(\frac{3}{2} \pi-\beta_{j}-\kappa_{j}-\theta\right)|z|^{-S} \text { in Cases IIIe and IIIf. }
\end{aligned}
$$

The proof of this lemma involves only elementary geometry, but is not entirely obvious. For illustration, we supply the proof in Cases IIIb and IIIf. For brevity, we denote ph $t$ by $\tau$.

Case IIIb. From (8.2), we derive

$$
\omega_{1}(z) \in\left[-\frac{1}{2} \pi, \frac{3}{2} \pi-\left(\beta_{j}-\alpha_{j}\right)-2 \kappa_{j}\right] \subseteq\left[-\frac{1}{2} \pi, 0\right],
$$

and

$$
\omega_{2}(z) \in\left[-\frac{3}{2} \pi+\beta_{j}-\alpha_{j}+2 \kappa_{j}, \frac{1}{2} \pi\right] \subseteq\left[0, \frac{1}{2} \pi\right] .
$$

Hence, from (9.10),

$$
\mathcal{V}_{\mathcal{P}_{1}(z)}\left(t^{-S}\right), \quad \mathcal{V}_{\mathcal{P}_{2}(z)}\left(t^{-S}\right) \leq \chi(S)|z|^{-S} .
$$

Similarly, when $t \in \mathcal{A}(z)$ and $-\pi-\alpha_{j}+\kappa_{j} \leq \tau \leq \pi-\beta_{j}-\kappa_{j}$, we have

$$
\nu_{\mathcal{P}_{1}(t)}\left(t^{-S}\right), \quad \nu_{\mathcal{P}_{2}(t)}\left(t^{-S}\right) \leq \chi(S)|t|^{-S} \leq \chi(S)|z|^{-S} .
$$

Next, if $t \in \mathcal{A}(z)$ and $\pi-\beta_{j}-\kappa_{j} \leq \tau \leq-\frac{1}{2} \pi-\alpha_{j}+\kappa_{j}$, then

$$
\omega_{2}(t) \equiv-\frac{1}{2} \pi-\alpha_{j}+\kappa_{j}-\tau \in\left[0,-\frac{3}{2} \pi+\beta_{j}-\alpha_{j}+2 \kappa_{j}\right] \subseteq\left[0, \frac{1}{2} \pi-2 \kappa_{j}\right] .
$$

Hence, (9.17) continues to apply in the case of $\mathcal{V}_{\mathcal{P}_{2}(t)}\left(t^{-S}\right)$. For $\mathcal{V}_{P_{1}(t)}\left(t^{-S}\right)$, let $t_{1}$ be the intersection of $\mathcal{P}_{1}(t)$ with ph $t=\pi-\beta_{j}-\kappa_{j}$; see Figure 8.2. Then from (9.11), we have

$$
\mathcal{V}_{\mathcal{P}_{1}(t)}\left(t^{-S}\right)<2 \chi(S)\left|t_{1}\right|^{-S} \leq 2 \chi(S)|z|^{-S} .
$$

The analysis in the remaining case, that is $t \in \mathcal{A}(z)$ and $\frac{1}{2} \pi-\beta_{j}-\kappa_{j} \leq \tau \leq$ $-\pi-\alpha_{j}+\kappa_{j}$, is similar.

Case IIIf. Let $z_{1}$ and $z_{1}^{\prime}$ be the intersections of $\mathcal{P}_{1}(z)$ with ph $t=\pi-\beta_{j}-\kappa_{j}$ and ph $t=-\pi-\alpha_{j}+\kappa_{j}$, respectively; see Figure 8.4. From (9.11), it is clear that if $t \in \mathcal{A}(z)$, then

$$
\mathcal{V}_{\mathcal{P}_{1}(t)}\left(t^{-S}\right)<2 \chi(S)\left|z_{1}\right|^{-S} \text {. }
$$

Next, suppose that $t \in \mathcal{A}(z)$ and $-\pi-\alpha_{j}+\kappa_{j} \leq \tau<\frac{3}{2} \pi-\beta_{j}-\kappa_{j}$. Then

$$
\omega_{2}(t) \in\left(-2 \pi+\beta_{j}-\alpha_{j}+2 \kappa_{j}, \frac{1}{2} \pi\right] \subseteq\left(-\frac{1}{2} \pi, \frac{1}{2} \pi\right] .
$$

Hence, from (9.10),

$$
\mathcal{V}_{\mathcal{P}_{2}(t)}\left(t^{-S}\right) \leq \chi(S)|t|^{-S} \leq \chi(S)\left|z_{1}\right|^{-S} .
$$

Alternatively, assume that $t \in \mathcal{A}(z)$ and $\frac{1}{2} \pi-\beta_{j}-\kappa_{j} \leq \tau \leq-\pi-\alpha_{j}+\kappa_{j}$. Then from (9.11),

$$
\mathcal{V}_{\mathcal{P}_{2}(t)}\left(t^{-S}\right)<2 \chi(S)\left|z_{1}^{\prime}\right|^{-S} \leq 2 \chi(S)\left|z_{1}\right|^{-S}
$$


Lastly, from Figure 8.4, we note that

$$
\left|z_{1}\right|=|z| \sin \left(\frac{3}{2} \pi-\beta_{j}-\kappa_{j}-\theta\right) .
$$

With the aid of Lemma 9.1 and the identities $\widehat{D}_{1 \ell}+\widehat{D}_{2 \ell}=D_{\ell}, \widehat{G}_{1}+\widehat{G}_{2}=G$, we now may construct bounds for $|R|$ in Case III analogous to those given by (9.13)-(9.16) in Cases I and II. They are as follows:

Case IIIa.

$$
|R| \leq \frac{\chi(S)}{1-\frac{1}{2} \pi G|z|^{-1}}, \quad|z|>\max \left(b, \frac{1}{2} \pi G\right)
$$

Case IIIb.

$$
|R| \leq \frac{2 \chi(S)}{1-\pi G|z|^{-1}}, \quad|z|>\max (b, \pi G) .
$$

Cases IIIc and IIId.

$$
\begin{aligned}
& |R| \leq \frac{2 \chi(S) \operatorname{cosec}^{S}\left(\theta+\frac{3}{2} \pi+\alpha_{j}-\kappa_{j}\right)}{1-\pi G \operatorname{cosec}\left(\theta+\frac{3}{2} \pi+\alpha_{j}-\kappa_{j}\right)|z|^{-1}}, \\
& |z|>\max (b, \pi G) \operatorname{cosec}\left(\theta+\frac{3}{2} \pi+\alpha_{j}-\kappa_{j}\right) .
\end{aligned}
$$

Cases IIIe and IIIf.

$$
\begin{aligned}
& |R| \leq \frac{2 \chi(S) \operatorname{cosec}^{S}\left(\frac{3}{2} \pi-\beta_{j}-\kappa_{j}-\theta\right)}{1-\pi G \operatorname{cosec}\left(\frac{3}{2} \pi-\beta_{j}-\kappa_{j}-\theta\right)|z|^{-1}} \\
& |z|>\max (b, \pi G) \operatorname{cosec}\left(\frac{3}{2} \pi-\beta_{j}-\kappa_{j}-\theta\right)
\end{aligned}
$$

\section{Completion of the proof of Theorems 3.1 and 3.2}

We now seek uniform $O$-estimates for the remainder term $R_{S j}(z)$ and its derivatives as $z \rightarrow \infty$ in the closed sector

$$
\mathcal{S}_{j}(\mathrm{~J}, \delta)=\left(z:-\frac{3}{2} \pi-\alpha_{j}+\delta \leq \mathrm{ph} z \leq \frac{3}{2} \pi-\beta_{j}-\delta\right) .
$$

Here $\delta$ is an arbitrary constant that we shall restrict to the interval

$$
0<\delta<\pi-\frac{1}{2}(\beta-\alpha)
$$

compare (3.6).

The constant $b$ introduced in $\S 6$ has to exceed both of the quantities $a$ and $\widehat{a}$ introduced in $\S 2$ and $\S 4$ and also satisfy $b \geq \nu_{j} \sqrt{2}$ where $\nu_{j}$ is defined by (6.11). At this stage, we restrict $b$ further, if necessary, by requiring

$$
b \geq \nu_{j} \max \left\{\sec \left(\frac{1}{4} \beta_{j}-\frac{1}{4} \alpha_{j}\right), \operatorname{cosec} \frac{1}{2} \delta\right\} .
$$

Then from (6.12), it follows that

$$
\beta_{j}-\alpha_{j} \leq 2 \pi-4 \kappa_{j}
$$

and

$$
2 \kappa_{j} \leq \delta
$$

The last inequality shows that

$$
\mathcal{S}_{j}(\mathcal{J}, \delta) \subseteq\left(z:-\frac{3}{2} \pi-\alpha_{j}+2 \kappa_{j} \leq \mathrm{ph} z \leq \frac{3}{2} \pi-\beta_{j}-2 \kappa_{j}\right) .
$$


Accordingly, if $z \in \mathcal{S}_{j}(\mathcal{J}, \delta)$ and $|z|$ is sufficiently large, then the conditions for the applicability of the results given in $\S 9$ for Cases I, II, and III are all satisfied. Furthermore,

$$
\begin{aligned}
\operatorname{cosec}\left(\theta+\frac{3}{2} \pi+\alpha_{j}-\kappa_{j}\right) & \leq \operatorname{cosec} \kappa_{j} \text { in Cases IIb, IIIc, and IIId, } \\
\operatorname{cosec}\left(\frac{3}{2} \pi-\beta_{j}-\kappa_{j}-\theta\right) & \leq \operatorname{cosec} \kappa_{j} \text { in Cases IIc, IIIe, and IIIf. }
\end{aligned}
$$

We conclude that if $z \rightarrow \infty$ in $\mathcal{S}_{j}(\mathcal{J}, \delta)$, then for each $\ell=0,1, \ldots, n-1$,

$$
\left(\frac{d}{d z}\right)^{\ell} R_{S j}(z)=e^{\lambda_{j} z} z^{\mu_{j}} O\left(z^{-S}\right)
$$

uniformly with respect to ph $z$. We observe also that the condition (7.1) can be removed for (10.6); in those cases in which $S \leq \operatorname{Re}\left(\mu_{j}-\mu_{k}\right)$, we may re-expand

$$
R_{S j}(z)=e^{\lambda_{j} z} z^{\mu_{j}} \sum_{s=S}^{S^{\prime}-1} \frac{a_{s j}}{z^{s}}+R_{S^{\prime} j}(z)
$$

where $S^{\prime}$ satisfies (7.1); compare (5.3).

In particular, with $\ell=0$, equation (10.6) shows that there is a solution of the differential equation (1.1) whose asymptotic expansion to $S$ terms within the sector $\mathcal{S}_{j}(\mathcal{J}, \delta)$ agrees with the formal expansion (2.11). However, we constructed this solution starting from (5.3) and (5.7), which suggests that it may depend on the choice of $S$. Denoting it by $w_{S j}(z)$, we have

$$
w_{S j}(z)=e^{\lambda_{j} z} z^{\mu_{j}}\left\{\sum_{s=0}^{S-1} \frac{a_{s j}}{z^{s}}+O\left(\frac{1}{z^{S}}\right)\right\}
$$

as $z \rightarrow \infty$ in $\mathcal{S}_{j}(\mathcal{J}, \delta)$. To complete the proof of Theorems 3.1 and 3.2 , we need to show that $w_{S j}(z)$ is independent of $S$, and also that the solution with the property (10.7) is unique.

We first observe that in the intersection

$$
\mathcal{S}(\mathcal{J}, \delta) \equiv\left\{z:-\frac{3}{2} \pi-\alpha+\delta \leq \operatorname{ph} z \leq \frac{3}{2} \pi-\beta-\delta\right\}
$$

of the sectors $\mathcal{S}_{j}(\mathcal{J}, \delta), j=1,2, \ldots, n$, we have a set of $n$ solutions $w_{S j}(z), j=$ $1,2, \ldots, n$, that enjoy the property $(10.7)$ for each $j$. We also know that (10.7) is $n-1$ times differentiable. On constructing the Wronskian of these solutions, we see that

$$
\begin{aligned}
& \mathcal{W}\left\{w_{S 1}(z), w_{S 2}(z), \ldots, w_{S n}(z)\right\} \\
& \quad=(\operatorname{det} \boldsymbol{\Lambda}) e^{\left(\lambda_{1}+\lambda_{2}+\cdots+\lambda_{n}\right) z} z^{\mu_{1}+\mu_{2}+\cdots+\mu_{n}}\left\{1+O\left(z^{-1}\right)\right\}
\end{aligned}
$$

as $z \rightarrow \infty$ in $\mathcal{S}(\mathcal{J}, \delta)$ where $\boldsymbol{\Lambda}$ is defined by (4.7). Since $\boldsymbol{\Lambda}$ is nonsingular, the $n$ solutions are linearly independent.

To prove that for each $j, w_{S j}(z)$ does not depend on the choice of $S$, we observe that as a consequence of $(10.2), \mathcal{S}(\mathcal{J}, \delta)$ exceeds half a Riemann sheet. Accordingly, the doubly-infinite line

$$
\operatorname{Re}\left\{\left(\lambda_{k}-\lambda_{j}\right) z\right\}=0, \quad k \neq j,
$$

divides the interior of $\mathcal{S}(\mathcal{J}, \delta)$ into either two or three open sectors. On at least one of these sectors, $\operatorname{Re}\left\{\left(\lambda_{k}-\lambda_{j}\right) z\right\}>0$, and on another, $\operatorname{Re}\left\{\left(\lambda_{k}-\lambda_{j}\right) z\right\}<0$. Let 
us denote the difference between any two solutions that are asymptotic to $e^{\lambda_{j} z} z^{\mu_{j}}$ as $z \rightarrow \infty$ in $\mathcal{S}(\mathcal{J}, \delta)$ by $\Delta w_{j}(z)$. Then

$$
\Delta w_{j}(z)=\sum_{k=1}^{n} A_{j k} w_{S k}(z)
$$

where the $A_{j k}$ are constants. When $k \neq j$, we may show that any one of these coefficients $A_{j \widehat{k}}$, say, vanishes, as follows. Let $z \rightarrow \infty$ along a ray $\mathcal{R}$ in $\mathcal{S}(\mathcal{J}, \delta)$ on which (i) $\operatorname{Re}\left\{\left(\lambda_{p}-\lambda_{q}\right) z\right\} \neq 0$ for all unequal values of $p$ and $q$, and (ii) $\operatorname{Re}\left\{\left(\lambda_{\widehat{k}}-\lambda_{j}\right) z\right\}>0$. It then follows that $A_{j k}=0$ for those values of $k$ for which $e^{\lambda_{k} z} z^{\mu_{k}}$ dominates $e^{\lambda_{j} z} z^{\mu_{j}}$ on $\mathcal{R}$. Since this includes $\widehat{k}$, we have $A_{j \widehat{k}}=0$. Accordingly, (10.9) reduces to

$$
\Delta w_{j}(z)=A_{j j} w_{S j}(z) .
$$

It then follows that $A_{j j}=0$ by letting $z \rightarrow \infty$ in $\mathcal{S}(\mathcal{J}, \delta)$. Thus $w_{S j}(z)=w_{j}(\mathcal{J} \mid z)$, say, where $w_{j}(\mathcal{J} \mid z)$ is independent of $S$.

This completes the proof of Theorems 3.1 and 3.2 .

\section{Summary and conclusions}

In this paper we have constructed new existence theorems for the asymptotic solutions of linear ordinary differential equations of arbitrary order in the neighborhood of an irregular singularity of rank 1 , on the assumption that the characteristic values are distinct. Advantages of the new theorems include more extensive regions of validity and availability of explicit bounds for the remainder terms. The theorems also will serve as a starting point for constructing hyperasymptotic expansions of the solutions on the lines of [9], [10], [12], [13], instead of the Borel-Laplace transform approach used in [11]. The only paper that has significant overlap with the present one is that of Stenger [19]. The theorems in this paper apply to systems of equations, rather than a single equation; more importantly, the regions of validity are not as extensive as those in the present paper, and the accompanying error bounds are not evaluated in explicit form. (On the other hand, Stenger's results are not restricted to singularities of unit rank nor to the case when the characteristic values are unequal.)

The asymptotic existence theorems are stated in $\S 3$ and proved in $\S \S 4-10$. In the proof, it becomes necessary to distinguish three cases, Case I, Case II, and Case III, as defined at the end of $\S 6$. Error bounds for these cases, expressed in terms of variations of negative powers of $z$ along certain straight-line paths in $\mathbb{C}$, are given by (7.10) in Case I, (7.12) and (7.14) in Case II, and (8.16) in Case III. Bounds for these variations are constructed in $\S 9$, leading to simpler bounds for the error terms, given by $(9.12)-(9.16)$ and $(9.18)-(9.21)$.

At this stage, two questions naturally arise.

First, are the new regions of validity given in $\S 3$ actually maximal? As a rule, the answer to this question is in the affirmative. This can be seen by analogy with the well-understood situation when $n=2$ [12], [15], [16, Chapter 7], as follows.

The sector of validity $\mathcal{S}_{j}(\mathcal{J})$ of the expansion $(3.9)$ of the solution $w_{j}(\mathcal{J} \mid z)$ always contains the nonempty closed sector

$$
\mathcal{T}_{j}(\mathcal{J})=\left(z:-\pi-\alpha_{j} \leq \operatorname{ph} z \leq \pi-\beta_{j}\right) .
$$

On each boundary of $\mathcal{T}_{j}(\mathcal{J})$, there is at least one solution $w_{k}(\mathcal{J} \mid z)$, say, over which $w_{j}(\mathcal{J} \mid z)$ achieves maximum dominance as $|z| \rightarrow \infty$, that is, the argument $\left(\lambda_{k}-\lambda_{j}\right) z$ 
of the function $\exp \left\{\left(\lambda_{k}-\lambda_{j}\right) z\right\}$ is real and negative. For example, on ph $z=\pi-\beta_{j}$, this is the solution, or solutions, for which $\theta_{j k}=\beta_{j}$. As ph $z$ increases or decreases without restriction, the form of the asymptotic expansion of $w_{j}(\mathcal{J} \mid z)$ keeps changing, in accordance with the Stokes phenomenon. In this way, a multiple of $w_{k}(\mathcal{J} \mid z)$ is introduced as ph $z$ crosses the corresponding boundary of $\mathcal{T}_{j}(\mathcal{J}) .{ }^{9}$ As ph $z$ continues to increase (or decrease), then at first $w_{j}(\mathcal{J} \mid z)$ continues to dominate $w_{k}(\mathcal{J} \mid z)$; accordingly, the contribution of the multiple of $w_{k}(\mathcal{J} \mid z)$ is absorbable in the $O$-estimates for the remainder terms associated with the expansion (3.9). However, this situation ends as soon as we reach the boundary of $\mathcal{S}_{j}(\mathcal{J})$ because on crossing this boundary the relative dominance of $e^{\lambda_{j} z} z^{\mu_{j}}$ and $e^{\lambda_{k} z} z^{\mu_{k}}$ is reversed. This means that unless the corresponding Stokes multiplier for $w_{k}(\mathcal{J} \mid z)$ is zero, the validity of (3.9) indeed does cease at the boundary of $\mathcal{S}_{j}(\mathcal{J})$.

The second question we address is: how sharp are the error bounds we have derived? Consider the most favorable situation first, Case I. In (9.12) and (9.13), the constants $B_{S j}, D_{\ell}$, and $G$ are defined in $\S \S 6-7$. By comparing (5.2)-(5.4) and (6.1), we see that when $b$ is large,

$$
B_{S j} \simeq\left|\phi_{0}^{\prime}\left(\lambda_{j}\right) a_{S j}\right|
$$

Similarly, from (6.2) and (6.3),

$$
D_{k \ell} \simeq\left|\lambda_{k}\right|^{\ell}
$$

and from (6.7)-(6.9),

$$
\widehat{C}_{k} \simeq\left|C_{k}\right|=1 /\left|\phi_{0}^{\prime}\left(\lambda_{k}\right)\right| .
$$

Substitution into (7.6a) then yields

$$
D_{\ell} \simeq \sum_{k=1}^{n} \frac{\left|\lambda_{k}\right|^{\ell}}{\left|\phi_{0}^{\prime}\left(\lambda_{k}\right)\right|}
$$

In (9.13), the actual value of $G$ is not critical since the factor $\exp \left(G|z|^{-1}\right)$ approaches 1 as $|z| \rightarrow \infty$. On combining the foregoing approximations, we see that if $a_{S j} \neq 0$, then for large $|z|$, the ratio of the error bound furnished by (9.12) and (9.13) to the asymptotic form of the actual error is asymptotic to

$$
\frac{\left|\phi_{0}^{\prime}\left(\lambda_{j}\right)\right|}{\left|\lambda_{j}\right|^{\ell}} \sum_{k=1}^{n} \frac{\left|\lambda_{k}\right|^{\ell}}{\left|\phi_{0}^{\prime}\left(\lambda_{k}\right)\right|}, \quad \ell=0,1, \ldots, n-1 .
$$

We may regard (11.1) as the overestimation factor for our bound. When $n=2$, this factor reduces to 2 when $\ell=0$ and to $\left(\left|\lambda_{1}\right|+\left|\lambda_{2}\right|\right) /\left|\lambda_{j}\right|$ when $\ell=1$ (provided that $\lambda_{j} \neq 0$ in the latter case). These values agree with those already available when $n=2$; compare [16, Chapter 7, Theorem 12.1]. We also observe that owing to the quantities $\left|\phi_{0}^{\prime}\left(\lambda_{k}\right)\right|^{-1}$ in (11.1), the overestimation factor will become large if any of the characteristic values are close together. This is to be expected since our results cease to apply when any of the $\lambda$ 's coalesce.

In Cases II and III, the asymptotic estimates of the error bounds (9.14), (9.18), and (9.19) contain extra factors of $\chi(S)$ or $2 \chi(S)$. Since $\chi(S) \sim\left(\frac{1}{2} \pi S\right)^{\frac{1}{2}}$ as $S \rightarrow \infty$, these factors increase the overestimation factors by a moderate amount. More severe are the effects of the cosecants raised to the power $S$ that appear in $(9.15),(9.16)$,

\footnotetext{
${ }^{9}$ See also [11, equation $\left.(2.13)\right]$, and [14, equation (8.7)].
} 
(9.20), and (9.21). However, as in the case $n=2$, this kind of deterioration is to be expected because we are approaching the boundaries of the regions of validity.

This concludes one part of the present investigation. Other parts are [11] and [14]. In [11], hyperasymptotic expansions are obtained for the solutions, but with smaller regions of validity than those furnished by Theorems 3.1 and 3.2. (However, as noted in [11], these regions can be increased by application of connection formulas.) In [14], we investigate more deeply the nature of the asymptotic solutions and develop methods for their computation for all values of $z$ and not merely in certain sectors in the neighborhood of the singularity. Both [11] and [14] also include new methods for computing the associated Stokes multipliers.

Acknowledgments. This research was stimulated by a workshop on Exponential Asymptotics held at the Isaac Newton Institute for Mathematical Sciences in Cambridge, U.K. from January 1995 through June 1995. The author is pleased to acknowledge the support of the Institute and of an EPSRC Visiting Fellowship for this period. He also thanks the referees for suggestions concerning the presentation of the results.

\section{References}

1. W. Balser, B. L. J. Braaksma, J.-P. Ramis, and Y. Sibuya, Multisummability of formal power series solutions of linear ordinary differential equations, Asymptotic Anal. 5 (1991), 27-45.

2. W. Balser, W. B. Jurkat, and D. A. Lutz, Birkhoff invariants and Stokes' multipliers for meromorphic linear differential equations, J. Math. Anal. Appl. 71 (1979), 48-94.

3. G. D. Birkhoff, Singular points of ordinary linear differential equations, Trans. Amer. Math. Soc. 10 (1909), 436-470.

4. J. C. Burkill, The Theory of Ordinary Differential Equations, Second edition, Oliver and Boyd, Edinburgh, 1962.

5. E. A. Coddington and N. Levinson, Theory of Ordinary Differential Equations, McGraw-Hill, New York, 1955.

6. T. M. Dunster, Error bounds for exponentially improved asymptotic solutions of ordinary differential equations having irregular singularities of rank one, Methods Appl. Anal. 3 (1996), 109-134.

7. E. L. Ince, Ordinary Differential Equations, Longmans, Green, London and New York, 1927. Reprinted by Dover, New York, 1956.

8. W. B. Jurkat, Meromorphe Differentialgleichungen, Lecture Notes in Mathematics No. 637, Springer-Verlag, Berlin, 1978.

9. B. T. M. Murphy and A. D. Wood, Hyperasymptotic solutions of second-order ordinary differential equations with a singularity of arbitrary integer rank, Methods Appl. Anal. [In press.]

10. A. B. Olde Daalhuis, Hyperasymptotic solutions of second-order linear differential equations II, Methods Appl. Anal. 2 (1995), 198-211.

11. H__ Hyperasymptotic solutions of higher order linear differential equations with a singularity of rank one, Proc. Roy. Soc. London Ser. A. [In press.]

12. A. B. Olde Daalhuis and F. W. J. Olver, Exponentially improved asymptotic solutions of ordinary differential equations. II Irregular singularities of rank one, Proc. Roy. Soc. London Ser. A 445 (1994), 39-56.

13. - Hyperasymptotic solutions of second-order linear differential equations I, Methods Appl. Anal. 2 (1995), 173-197.

14. On the asymptotic and numerical solution of linear ordinary differential equations, SIAM Rev. 40 (1998) [In press.]

15. F. W. J. Olver, On the asymptotic solutions of second-order differential equations having an irregular singularity of rank one, with an application to Whittaker functions, SIAM J. Numer. Anal. Ser. B 2 (1965), 225-243.

16. __ Asymptotics and Special Functions, Academic Press, New York, 1974. Reprinted by AK Peters, Wellesley, 1997. 
17. Asymptotic expansions of the coefficients in asymptotic series solutions of linear differential equations, Methods Appl. Anal. 1 (1994), 1-13.

18. F. W. J. Olver and F. Stenger, Error bounds for asymptotic solutions of second-order differential equations having an irregular singularity of arbitrary rank, SIAM J. Numer. Anal. Ser. B 2 (1965), 244-249.

19. F. Stenger, Error bounds for asymptotic solutions of differential equations $I$. The distinct eigenvalue case, J. Res. Nat. Bur. Standards Sect. B 70 (1966), 167-186.

20. Error bounds for asymptotic solutions of differential equations. II. The general case, J. Res. Nat. Bur. Standards Sect. B 70 (1966), 187-210.

21. B. Yu. Sternin and V. E. Shatalov, Borel-Laplace Transform and Asymptotic Theory: Introduction to Resurgent Analysis, CRC Press, Boca Raton, 1996.

22. W. J. Trjitzinsky, Analytic theory of linear differential equations, Acta. Math. 62 (1934), 167226.

23. Laplace integrals and factorial series in the theory of linear differential and linear difference equations, Trans. Amer. Math. Soc. 37 (1935), 80-146.

24. H. L. Turrittin, Convergent solutions of ordinary linear homogeneous differential equations in the neighborhood of an irregular singular point, Acta. Math. 93 (1955), 27-66.

25. V. S. Varadarajan, Linear meromorphic differential equations: a modern point of view, Bull. Amer. Math. Soc. (N.S.) 33 (1996), 1-42.

26. W. Wasow, Asymptotic Expansions for Ordinary Differential Equations, Wiley (Interscience), New York, 1965. Reprinted by Krieger, Huntington, 1976 and Dover, New York, 1987.

27. J. H. Wilkinson, The Algebraic Eigenvalue Problem, Clarendon Press, Oxford, 1965.

Institute for Physical Science and Technology and Department of Mathematics, UniverSity of Maryland, College Park, Maryland 20742, U.S.A.

E-mail: olver@bessel.umd.edu 